\title{
Looking for inhibitors of the dengue virus NS5 RNA-dependent RNA-polymerase using a molecular docking approach
}

This article was published in the following Dove Press journal:

Drug Design, Development and Therapy

II October 2016

Number of times this article has been viewed

\author{
Vicente Galiano' \\ Pablo Garcia-Valtanen ${ }^{2}$ \\ Vicente $\mathrm{Micol}^{3,4}$ \\ José Antonio Encinar ${ }^{3}$ \\ 'Physics and Computer Architecture \\ Department, Miguel Hernández \\ University (UMH), Elche, Spain; \\ ${ }^{2}$ Experimental Therapeutics \\ Laboratory, Hanson and Sansom \\ Institute for Health Research, School \\ of Pharmacy and Medical Science, \\ University of South Australia, Adelaide, \\ Australia; ${ }^{3}$ Molecular and Cell Biology \\ Institute, Miguel Hernández University \\ (UMH), Elche, Spain; ${ }^{4} \mathrm{CIBER}$ : \\ CBI 2/03/30038, Physiopathology of \\ the Obesity and Nutrition, CIBERobn, \\ Instituto de Salud Carlos III, Palma de \\ Mallorca, Spain
}

\begin{abstract}
The dengue virus (DENV) nonstructural protein 5 (NS5) contains both an N-terminal methyltransferase domain and a C-terminal RNA-dependent RNA polymerase domain. Polymerase activity is responsible for viral RNA synthesis by a de novo initiation mechanism and represents an attractive target for antiviral therapy. The incidence of DENV has grown rapidly and it is now estimated that half of the human population is at risk of becoming infected with this virus. Despite this, there are no effective drugs to treat DENV infections. The present in silico study aimed at finding new inhibitors of the NS5 RNA-dependent RNA polymerase of the four serotypes of DENV. We used a chemical library comprising 372,792 nonnucleotide compounds (around 325,319 natural compounds) to perform molecular docking experiments against a binding site of the RNA template tunnel of the virus polymerase. Compounds with high negative free energy variation $(\Delta \mathrm{G}<-10.5 \mathrm{kcal} / \mathrm{mol})$ were selected as putative inhibitors. Additional filters for favorable druggability and good absorption, distribution, metabolism, excretion, and toxicity were applied. Finally, after the screening process was completed, we identified 39 compounds as lead DENV polymerase inhibitor candidates. Potentially, these compounds could act as efficient DENV polymerase inhibitors in vitro and in vivo.
\end{abstract}

Keywords: virtual screening, molecular docking, high-throughput computing, AutoDock/Vina, ADMET, SuperNatural database, inhibitors, NS5 RNA-dependent RNA polymerase

\section{Introduction}

Members of the Flaviviridae family cause a large variety of diseases in humans and other animal species. Flaviviruses can be transmitted from animals to humans by arthropod vector species such as ticks and mosquitoes. For example, the mosquito Aedes aegypti can transmit the chikungunya, yellow fever, and Zika viruses. ${ }^{1}$ Also, humans can be infected by contact with infected blood. ${ }^{2}$ The family Flaviviridae includes four main genera, Flavivirus, Pestivirus, Hepacivirus, and Pegivirus, as well as some unclassified viruses. ${ }^{3}$ The genus Flavivirus comprises 67 viruses, several of which infect humans, for instance, dengue virus (DENV), Japanese encephalitis virus, yellow fever virus, West Nile virus, and tick-borne encephalitis virus. ${ }^{2}$ DENV poses a major risk for human health. It is estimated that half of the population of the world is at risk of becoming infected with one serotype of DENV or another. ${ }^{4}$ Recent data indicated that in 2013, DENV caused 40-58 million symptomatic infections, including 13,586 fatal cases, with an associated financial cost of US\$ 8.9 billion. ${ }^{5}$ DENV infection in humans develops differently in each case. Globally, in 2013, 18\% of DENV-infected patients were admitted to hospital, $48 \%$ received medical attention or advise outside a hospital, and $34 \%$ did not need, find, or seek medical attention. ${ }^{5}$ Clinical manifestations
Correspondence: José Antonio Encinar Molecular and Cell Biology Institute, Miguel Hernández University, Edificio Torregaitán, Avenida de la Universidad, Elche, Spain

Tel +3496658453

Fax +34966658758

Email jant.encinar@umh.es
Drug Design, Development and Therapy 2016:10 3163-3181

3163 
of DENV disease range from mild dengue fever to severe dengue hemorrhagic fever and dengue shock syndrome. In late 2015 and early 2016, the first dengue vaccine, Dengvaxia (CYD-TDV), manufactured by Sanofi Pasteur (Lyon cedex, France), was registered in several countries for its use in individuals aged between 9 and 45 and living in DENV-endemic areas. ${ }^{6}$ However, despite its huge impact on public health around the world, effective antiviral therapies against DENV and other flaviviruses have not been developed yet.

The DENV virus genome is made up of a single strand of positive-sense (ie, it is directly translated into protein) RNA, which is copied in endoplasmic reticulum membraneassociated replication complexes in the host cell. Such complexes contain the DENV nonstructural proteins 3 and 5 (NS3 and NS5) along with host proteins. ${ }^{7}$ The genomic RNA contains a $5^{\prime}$ untranslated region, a single open reading frame, and another $3^{\prime}$ untranslated region. The open reading frame encodes a polyprotein that is processed by viral and host proteases generating ten mature viral proteins: three structural proteins (capsid [C], membrane [M], and envelope [E]) and seven nonstructural proteins (NS1, NS2A, NS2B, NS3, NS4A, NS4B, and NS5). ${ }^{8}$

NS5 (900 amino acids) exerts three different enzymatic activities. ${ }^{9}$ The MTase activity protects viral mRNA from degradation by 5 '-exoribonucleases and ensures their recognition by eukaryotic translation initiation factor. ${ }^{10}$ The N-terminal MTase domain (residues 1-262, guanosine-5' triphosphate-binding site, $S$-adenosylmethionine binding site, and NS3 protease interacting site) is connected through a ten-residue linker to the C-terminal RNA-dependent RNA polymerase (RdRp) domain formed by residues $273-900 .{ }^{11}$ In turn, the RdRp domain is formed by three subdomains called fingers (273-315, 416-496, and 543-600), palm (497-542, 601-705), and thumb (706-900). ${ }^{12}$ The active center of the enzyme is defined by the conserved GDD protein motif located in the palm domain. The amino acid sequence between residues 320 and 405 includes two nuclear localization sequences that are recognized by cellular factors, allowing protein transport to the nucleus. In conjunction with other viral proteins and host cell proteins, the NS5 polymerase domain promotes the formation of double-stranded RNA intermediates, both positive and negative sense. Negativesense strands serve as templates for the DENV RdRp to synthesize new positive-sense genomic RNA. ${ }^{13,14}$

RNA viruses, such as DENV, are the only organisms that possess enzymes with RdRp activity that is essential for their replication. The lack of RdRps in human cells as wells as in other animal species makes these polymerases an attractive antiviral target. ${ }^{15}$ Current polymerase inhibitors belong to one of two types of molecules: first, nucleoside/nucleotide analogs (NIs) that function as RNA chain terminators ${ }^{16}$ and second, nonnucleoside inhibitors which bind to different sites of the catalytic binding site of the enzyme, blocking the conformational switch from polymerase initiation to elongation or impeding the processivity of polymerase elongation. ${ }^{17}$ In the literature, we can find examples of shortfalls and side effects of some of the NIs against the DENV polymerase. ${ }^{18}$ Sometimes due to the lack of selectivity, NIs may have off-target effects on other polymerases such as mitochondrial DNA polymerase- $\gamma$, resulting in mitochondrial toxicity. ${ }^{19,20}$ The first reported allosteric inhibitor of DENV RdRp (NITD-1 compound, $\mathrm{IC}_{50}=7.2 \mu \mathrm{M}$ ) was identified by means of high-throughput screening using a primer extension-based assay; the second was modified at the $N$-sulfonylanthranilic acid lead (NITD-2 compound, $\left.\mathrm{IC}_{50}=0.7 \mu \mathrm{M}\right)^{21}$ to selectively inhibit DENV-2 polymerase. NITD-29, a photoreactive derivative of NITD-2 $\left(\mathrm{IC}_{50}=1.5 \mu \mathrm{M}\right)$, has been used to map the compoundbinding site on the protein and, therefore, define its regulatory allosteric site. ${ }^{21}$ Mass spectrometry of the ultraviolet cross-linked NITD-29/NS5 complex and molecular docking simulations have helped to further define the allosteric binding pocket near the residues Arg737, Thr413, and Met343 located at the RNA template tunnel. Thus, in light of these findings, it could be assumed that the inhibitory effect of these compounds is due to their ability to compete with the RNA template in the RNA template tunnel. ${ }^{21}$ Unfortunately, these three compounds are inactive in cell culture. ${ }^{21}$

In this context, we performed an in silico study to find new potential NS5 RdRp efficient agonists from a chemical library built with molecules sharing $\geq 70 \%$ structural similarity with NITD-1, NITD-2, and NITD-29 ( 47,473 compounds recorded in the PubChem database $\left.{ }^{22}\right)$ together with another chemical library of natural products $(\sim 325,319$ compounds recorded in the SuperNatural II database ${ }^{23}$ ). The best scoring compounds were compared with NITD inhibitors based on their ability to bind to DENV NS5 RdRp at the RNA template tunnel. In addition to this, compounds that showed the best binding scores were also evaluated for their pharmacodynamics, pharmacokinetics, and toxicity properties. At the end of the study, 39 compounds passed the selection process. These compounds scored better than NITD-1, NITD-2, and NITD-29, which suggests that these 39 compounds could overcome some of the problems experienced with NITD molecules, such as their inability to inhibit NS5 RdRp in live cell systems. Our in silico approach 
provides the basis for subsequent in vitro and in vivo studies to test these newly identified DENV NS5 RdRp inhibitor candidates.

\section{Materials and methods Protein structures for Dengue NS5 RdRp and chemical libraries}

To date, several crystal structures of DENV-3 NS5 RdRd protein have been solved and deposited in the Protein Data Bank (4V0Q, ${ }^{11} 4 \mathrm{~V} 0 \mathrm{R},{ }^{11}$ 5DTO $,{ }^{24} 2 \mathrm{~J} 7 \mathrm{U},{ }^{12} 2 \mathrm{~J} 7 \mathrm{~W},{ }^{12} 3 \mathrm{VWS},{ }^{25}$ $\left.4 \mathrm{C} 11,{ }^{26} 4 \mathrm{HHJ}^{25}\right)$. However, in all structures, several residues were missing. Herein, the three-dimensional (3D) atomic coordinates for these amino acid sequences were obtained by constructing a model, using a homology modeling approach, for the RdRps of each of the four serotypes of DENV. Three 3D theoretical models of each UniProt dengue NS5 RdRp were built at Swiss-Model server ${ }^{27}$ using the crystallographic structures of 4C11, 3VWS, 4V0Q, and 5DTO (Table 1) as templates. Arg737, Thr413, and Met343 or equivalent positions in the different protein sequences have been used

Table I Protein sequences used in the homology modeling of the NS5 RdRp for all four dengue virus serotypes

\begin{tabular}{|c|c|c|c|c|}
\hline Serotype & UniProt - RdRp & $\begin{array}{l}\text { Structures of NS5 RdRp } \\
\text { at Protein Data Bank }\end{array}$ & $\begin{array}{l}\text { NS5 RdRp } \\
\text { homology model }\end{array}$ & $\begin{array}{l}\text { PDB template } \\
\text { for homology } \\
\text { modeling }\end{array}$ \\
\hline \multirow[t]{6}{*}{ DENV-I } & POLG_DENIB P27909/2733-3392 & None & POLG_DENIB-I.pdb & $4 \mathrm{CII}$ \\
\hline & & & POLG_DENIB-2.pdb & 3VWS \\
\hline & & & POLG_DENIB-3.pdb & $4 \mathrm{Cll}$ \\
\hline & POLG_DENIW PI7763|2733-3392 & None & POLG_DENIW-I.pdb & $4 \mathrm{CII}$ \\
\hline & & & POLG_DENIW-2.pdb & 3VWS \\
\hline & & & POLG_DENIW-3.pdb & $4 \mathrm{Cll}$ \\
\hline \multirow[t]{18}{*}{ DENV-2 } & POLG_DEN2J P07564|2732-339| & None & POLG_DEN2J-1.pdb & $4 \mathrm{Cll}$ \\
\hline & & & POLG_DEN2J-2.pdb & $4 \mathrm{ClI}$ \\
\hline & & & POLG_DEN2J-3.pdb & 3VWS \\
\hline & POLG_DEN2N PI4340|2732-339| & None & POLG_DEN2N-I.pdb & $4 \mathrm{Cll}$ \\
\hline & & & POLG_DEN2N-2.pdb & $4 \mathrm{CII}$ \\
\hline & & & POLG_DEN2N-3.pdb & 3VWS \\
\hline & POLG_DEN2P PI 2823/2729-3388 & None & POLG_DEN2P-I.pdb & $4 \mathrm{CII}$ \\
\hline & & & POLG_DEN2P-2.pdb & 3VWS \\
\hline & & & POLG_DEN2P-3.pdb & $4 \mathrm{CII}$ \\
\hline & POLG_DEN26 P29990|2732-339| & None & POLG_DEN26-I.pdb & $4 \mathrm{CII}$ \\
\hline & & & POLG_DEN26-2.pdb & $4 \mathrm{CII}$ \\
\hline & & & POLG_DEN26-3.pdb & 3VWS \\
\hline & POLG_DEN27 P2999||2732-339| & None & POLG_DEN27-I.pdb & $4 \mathrm{CII}$ \\
\hline & & & POLG_DEN27-2.pdb & $4 \mathrm{ClI}$ \\
\hline & & & POLG_DEN27-3.pdb & 3VWS \\
\hline & POLG_DEN28 PI4337|2732-339| & None & POLG_DEN28-I.pdb & $4 \mathrm{CII}$ \\
\hline & & & POLG_DEN28-2.pdb & $4 \mathrm{ClI}$ \\
\hline & & & POLG_DEN28-3.pdb & 3VWS \\
\hline \multirow[t]{6}{*}{ DENV-3 } & POLG_DEN3I Q5UB51|2730-3390 & 4V0Q, 4V0R, 5DTO & POLG_DEN3-I.pdb & $4 \mathrm{~V} 0 \mathrm{Q}$ \\
\hline & & & POLG_DEN3-2.pdb & $4 \mathrm{VOR}$ \\
\hline & & & POLG_DEN3-3.pdb & 5DTO \\
\hline & Q6DLV0_9FLAV Q6DLV0|2730-3390 & $2 \mathrm{~J} 7 \mathrm{U}, 2 \mathrm{~J} 7 \mathrm{~W}, 3 \mathrm{VWS}, 4 \mathrm{CII}, 4 \mathrm{HHJ}$ & Q6DLV0 9FLAV-I.pdb & 5DTO \\
\hline & & & Q6DLV0_9FLAV-2.pdb & $4 \mathrm{VOR}$ \\
\hline & & & Q6DLV0_9FLAV-3.pdb & $4 \mathrm{VOR}$ \\
\hline \multirow[t]{9}{*}{ DENV-4 } & POLG_DEN4P Q58HT7|2728-3387 & None & POLG_DEN4D-I.pdb & $4 \mathrm{CII}$ \\
\hline & & & POLG_DEN4D-2.pdb & $4 \mathrm{VOR}$ \\
\hline & & & POLG_DEN4D-3.pdb & $4 \mathrm{~V} 0 \mathrm{Q}$ \\
\hline & POLG_DEN4D P09866|2728-3387 & None & POLG_DEN4P-I.pdb & $4 \mathrm{CII}$ \\
\hline & & & POLG_DEN4P-2.pdb & $4 \mathrm{VOR}$ \\
\hline & & & POLG_DEN4P-3.pdb & $4 \mathrm{~V} 0 \mathrm{Q}$ \\
\hline & POLG_DEN4T Q2YHF0|2728-3387 & None & POLG_DEN4T-I.pdb & 5DTO \\
\hline & & & POLG_DEN4T-2.pdb & $4 \mathrm{VOR}$ \\
\hline & & & POLG_DEN4T-3.pdb & $4 \mathrm{CII}$ \\
\hline
\end{tabular}

Notes: UniProt code includes information of the number of aminoacids for the polymerase into the full-length polyprotein. Models are available at http://docking.umh.es/ Abbreviations: NS5, nonstructural protein 5; RdRp, RNA-dependent RNA polymerase; PDB, Protein Data Bank. 
to define the ligand-binding site. ${ }^{21}$ For each model, water molecules, ions, or inhibitors were removed.

The two-dimensional (2D) structures of 47,473 compounds from the PubChem database ${ }^{22}$ and 325,319 molecules of natural compounds from the SuperNatural II database ${ }^{23}$ were downloaded in spatial data file format. To handle the large number of ligand structures, we used a script ${ }^{28}$ to convert 2D spatial data files into individual 3D structures in mol2 format using Marvin Suite 6.0 from ChemAxon (http:// www.chemaxon.com). Mol2 files were converted into pdbqt format using the Python script "prepare_ligand4.py" included in the AutoDockTools-1.5.7.rc1. ${ }^{29}$

\section{Docking procedure}

Prior to initiating the docking procedure, the protein (receptor) and ligand structures should be prepared. ${ }^{28}$ All of the NS5 RdRp modeled protein structures were then subjected to geometry optimization using the repair function of the FoldX algorithm. ${ }^{30}$ We perform docking with AutoDock/Vina ${ }^{31}$ using a grid with dimensions of $24 \times 24 \times 24$ points centered to residues Arg737, Thr413, and Met343 or equivalent positions in the different protein sequences. AutoDock/Vina was set up on a Linux cluster at calendula.fcsc.es (Linux cluster ROCKS 6.1 distribution) (Castilla y Leon Supercomputing Center), lusitania2.cenits.es Linux cluster (Research, Technological Innovation and Supercomputing Center of Extremadura [CenitS]), and login-hpc.ceta-ciemat.es Linux cluster (Extremadura Research Centre for Advanced Technologies [CETA-CIEMAT]). Compounds with the lowest calculated free energy variations (ie, the best theoretical binding energy) were selected as putative modulators.

\section{In silico analysis of pharmacokinetic parameters and toxicity potential properties}

Molecular descriptors, such as the topological polar surface area (TPSA), molecular weight (MW), the estimated logarithm (base 10) of the solubility measured in mol/L $(\operatorname{logS})$, the estimated logarithm (base 10) of the partition coefficient between $n$-octanol and water $(\log \mathrm{P})$, the number of hydrogen bond acceptors, the number of hydrogen bond donors, the violations of Lipinski's rule of five ${ }^{32}$ (Ro5 violations), the drug score (DrugScore), and the fragment-based druglikeness (Druglikeness), were calculated using DataWarrior v4.2.2 software (Allschwil, Switzerland). ${ }^{33}$ The in silico absorption, distribution, metabolism, excretion, and toxicity (ADMET) properties of all compounds were calculated using admet$\mathrm{SAR}^{34}$ and DataWarrior v.4.2.2 software (mutagenicity, tumorigenicity, irritancy, and reproductive effects).

\section{Results and discussion Homology modeling of NS5 RdRp for all DENV serotypes}

The DENV-3 NS5 RdRp X-ray crystallographic structure is the only available in Protein Data Bank (Table 1). Three structures exist for the sequence POLG_DEN3I (UniProt code Q5UB51, sequence 2,730-3,390), that is, 4V0Q, 4V0R, and 5DTO, and five for Q6DLV0_9FLAV (UniProt code Q6DLV0, 2,730-3,390), that is, 2J7U, 2J7W, 3VWS, $4 \mathrm{C} 11$, and $4 \mathrm{HHJ}$. None of these structures include all of the residues in RdRp domain. For example, 4V0Q does not include residues 405-415; 453-467, 4V0R residues 404-415; 453-466, and 5DTO residues 405-414; 453-468. Likewise, 2J7U does not contain residues 309-314; 404-416; 449-467, 2J7W residues 308-314; 406-417; 449-469, 3VWS residues 312-315; 342-348; 454-462, 4C11 residues 405-415; 453-470, and 4HHJ residues 405-417; 455-466. In all cases, the unresolved residues are located in the RNA template tunnel, that is, the target site of our molecular docking experiments. Therefore, we generated the structural models for the NS5 RdRp protein sequences of DENV-3 as well as for the other three serotypes DENV-1, -2, and -4 (Table 1).

The protein sequences of NS5 RdRp of DENV-1 to -4 are highly conserved, with a minimum identity of $75 \%$ (Figure 1). The sequences close to the amino acids that define the allosteric binding proteins (Arg737, Thr413, and Met343) are especially conserved, except near the DENV-3 Thr413 site. The results of Niyomrattanakit et al strongly indicate that NITD-29 was cross-linked to Met343 within the RdRp domain of NS5 and the sequence alignment showed that this position is absolutely conserved among the four serotypes of DENV. Docking and molecular simulation suggest that the compound binds to the RNA template formed between the finger and thumb subdomains. ${ }^{21}$ Arg737 is conserved among mosquito-borne flavivirus NS5. NITD-29 compound inhibits RdRp activity through competing with RNA template in the RNA template tunnel. ${ }^{21} \mathrm{With}$ these sequences, three 3D models were generated (Table 1; Figure 1) by homology modeling (in automated mode ${ }^{27}$ ) as the first step to perform molecular docking experiments.

\section{NS5 RdRp-ligand molecular docking analysis}

The "induced-fit" Koshland theory states that the active site of the protein is continually reshaped by interactions with the ligands, as the ligands interact with the protein. Therefore, ligand and receptor should be treated as flexible during molecular docking. ${ }^{35}$ However, due to limitations in the 
Q2YHFO I DENV 4 MTI I GRRLQRLQEEHKETWHYDHENPYRT WAYHGSYEAPST GSASSMVNGVVKLLTKPWD P09866 I DENV 4 MT I I GRRLQRLQEEHKETWHYDQENPYRTWAYH GSYEAPST GSASSMVNGVVKLLTTKPWD P12823|DENV2 IDIIGKRIEKIKOEHET SWHYDQDHPYKTWAYHGSYETKOTGSASSMVNGVVRLITKPWD P07564 | DENV2 IDIIGKRIEKIKOEHETSWHYDQDHPYKTWAYHGSYETKOTGSASSMVNGVVRLLTKPWD P29991 | DENV2 IDIIGKRIEKI KQEHET SWHYDQDHPYKTWAYHGSYETKQT GSASSMVNGVERLITKPWD P29990 I DENV2 LDIIGKRIEKIKOEHET SWHYDQDHPYKTWAYHGSYETKOTGSASSMVNGVRRLLTKPWD P14340 | DENV2 LDIIGKRIEKIKQEHETSWHYDQDHPYKTWAYHGSYETKQTGSASSMGNGVVRLLTKPWD P14337 | DENV2 LDIIGKRIEKI KQEHET SWHY DQDHPYKTWAYHGSYETKQT GSASSMVNGVVRLLTKPWD P27909 | DENV1 IDIIGQRI ENI KNEHKSTWHYDEDNPYKTWAYHGSYEVKPSGSASSMVNGVVRLLTKPWD P17763 I DENV1 IDI I GQRI ENI I KNGHKSTWHYDEDNPYKTWAYHGGSYEVKP SGSASSMVNGVVRLLTKPWD Q6DLVO DENV 3 MDVIGERIKRIKEEHNSTWHYDDENPY KTWAYHGSYEVKATGSASSMINGVVKLLTKPWD

Q2YHFO IDENV4 CTREEFISKVRSNAAIGAVFQEEQGWT SASEAVNDSRFWELVDKERALHOEGKCESCVYN 100 P09866 | DENV4 CTREEFISKVRSNAAIGAVFOEEQGWTSASEAVNDSRFWELVDKERALHOEGKCESCVYN Q58HT7 I DENV4 CTREEFISKVRSNAAI GAVFQEEQGWT SASEAVNDSRFWELVDKERALHQEGKCESCVYN P12823 I DENV2 CTREEETKKVRSNAALGAI FTDENKWKSAREAVEDSRFWELVDKERNLHLEGKCETCVYN P29991 I DENV2 CTREEFTRKVRSNAALGAI FTDENKWKSAREAVEDSR FWELVDKERNLHLEGKCETCVYN P29990 I DENV2 CTREEFTRKVRSNAALGAI FTDENKWKSAREAVEDSRFWELVDKERNLHLEGKCETCVYN P14340 | DENV2 2 CTREEFTRKVRSNAALGAI FTDENKWKSAREAVEDSRFWELVDKERNLHLEGKCETCVYN
P14337 IDENV2 CTREEFTRKVRSNAALGAI FT DENKWKSAREAVEDRRFWELVDKERNLHLEGKCETCVYN P14337 I DENV2 CTREEFTRKVRSNAALGAI FT DENKWKSAREAVEDSRFWELVDKERNLHLEGKCETCVYN
P27909 DENV 1 CTREEFTRKVRSNAAI GAVFVDENOWNSAKEAVEDERFDLVHRERELHKOGKCATCVYN P27909 | DENV1 CTREEFTRKVRSNAAIGAVVVDENQWNSAKEAVEDER FWDLVHRERELHKQGKCATCVYN
P17763 | DENV1 CTREETTRKRSNAAIGAVEVDENQWNSAKEAVEDERFWDLVHRERELHKQGKCATCVYN P17763 | DENV1 CTREEFTRKVRSNAAIGAVFVDENQWNSAKEAVEDER FWDLVHRERELHKQGKCATCVYN
Q5UB51 | DENV3 CTREEFTKKVRTNAAMGAVFTEENQWDSARAAVEDEE FWKLVDRERELHKLGKCGSCVYN Q5UB51 IDEN3 CTREEFTKKVRTNAAMGAVFTEENQWDSARAAVEDEE FWKLVDRERELHKLGKCGSCVYN$$
{ }^{*} \dot{4} \dot{4} 13
$$

Q2YHEO |DENV4 RLGYILEDIDKKDGDLIYADDTAGWDTRITEDDLLNEELITEQMAPHHKILAKAIFKLTY P09866 I DENV4 RLGYILEEIDKKDGDLMYADDTAGWDTRIT EDDLQNEELITEQMAPHHKILAKAI FKLTY
O58HT7 | DENV4 RLGYILEDIDKKDGDLIYADDTAGWDTRITEDDLINEELITEOMAPHHKILAKAIFKLTY Q58HT7 | DENV4 RLGYILEDIDKKDGDLIYADDTAGWDTRIT EDDLINEELITEQMAPHHKILAKAI FKLTY
P12823 | DENV2 KLGYILREVSKKEGGAMYADDTAGWDTRITIEDLKNEEMITNHMAGEHKKLAEAIFKLTY P12823 | DENV2 KLGYILREVSKKEGGAMYADDTAGWDTRITI EDLKNEEMI TNHMAGEHKKLAEAI FKLTY
P07564 | DENV2 KLGYILRDVSKKEGGAMYADDTAGWDTRITLEDLKNEEMVTNHMEGEHKKLAEAIFKLTY P07564 | DENV2 KLGYILRDVSKKEGGAMYADDTAGWDTRITLEDLKNEEMVTNHMEGEHKKLAEAIFKLTY
P29991 | DENV2 KLGYILRDVSKKEGGAMYADDTAGWDTRITLEDLKNEAMVTNHMEGEHKLAEAIFKLTY P29990 | DENV2 KLGYILRDVSKKEGGAMYADDTAGWDTRITLEDLKNEEMVTNHMEGEHKKLAEAI FKLTY P14340 | DENV2 KLGYILRDVSKKEGGAMYADDTAGNDTRITLEDLKNEEMVTNHMEGEHKKLAEAI FKLTY P14337 | DENV2 KLGYILRDVSKKEGGAMYADDTAGWDTRITLEDLKNEEMVTNHMEGEHKKLAEAI FKLTY P27909|DENV1 KLGYILRDISKIPGGNMYADDTAGNDTRITEDDLQNEAKITDIMEPEHALLATSIFKLTY P17763 DENV1 KLGYILRDISKI PGGNMYADDTAGWDTRITEDDLONEAKITDIMEPEHALLATSIFKLTY Q6DLVO IDENV 3 KLGYILRDISKI PGGAMYADDTAGWDTRITEDDLHNEEKI IQQMDPEHRQLANAIFKLTY

GDD motif

Q2YHFO I DENV 4 MHNPKGLKERVEKWLKECGVDRLKRMAISGDDCVVKPLDERESTSLLELLDMGKVRKDIP P09866 I DENV 4 MQNPKGLKERVEKWLKECGVDRLKRMAISGDDCVVKPIDERFGTSLI ILNDMGKVRKDIP

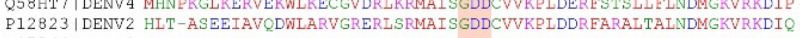
P07564 I DENV2 HLT-VTEEIAVQNWLARVGRERLSRMAISGDDCVVKPIDDRFASALTALNDMGKVRKDIO P29991 | DENV2 HLT-ITEEIAVQNWL_ARVGRERLSRMAISGDDCVVKPLDDRLPSALTALNDT GKIRKDIO P29990 | DENV2 HLT-ITEEIAVQNWLARVGRERLSRMAISGDDCVVKPLDDRLPSALTALNDMGKIRKDIQ P14340 | DENV2 HLT-VT EEIAVQNWLARVGRERLSRMAISGDDCVVKPLDDRREASALTALNDMGKVRKDIO P14337 | DENV2 HLT-VT EEIAVQNWLARVGRERLSRMAISGDDCVVKPLDDRFASALTTALNDMGKIRKDIQ P27909 | DENV1 IESPNL-AERVLDWLEKHGAERLKRMAISGDDCVVKPIDDRFATALIALNDMGKVRKDII
P17763 | DENV1 IET PNL-AERVLDWLKKHGTERLKRMAISGDDCVVKPIDDRFATALTALNDMGKVRKDI Q5UB51 | DENV3 LENPHPLEKKI TOWLETKGVERLKRMAISGDDCVVRPIDDRFANALIALNDMGKVRKDI Q6DLVO I DENV3 LENPHLLEKKI TQWLETKGVERLKRMAISGDDCVVKPIDDRANALIALNDMGKVRKDIP

Q2YHFO I DENV4 CLGKAYAQMWSLMY FHRRDLRLASMAICSAVPTEWFPT SRTTWSIHAHHOWMTTEDMLKV P09866 I DENV 4 CLGKAYAQMWSLMY FHRRDLRLASMAICSAVPTEWFPTSRTTWSIHAHHQWMTTEDMLKV Q58HT7 | DENV 4 CLGKAYAQMWSLMY FHRRDLRLA.SMAICSAVPTEWFPT SRTTWSIHAHHQWMTTEDMLKV
P12823 | DENV2 CLGKSYAOMWSIMY FHRRDLRLAANAICSAVPSHWVPTSRTTWSIHA.SHEWMTTEDMLTV P12823 | DENV2 2 CLGKSYAOMWSLMY FHRRDLRLAANAICSAVP SHWVPTSRTTWSIHASHEWMTTEDMLTV
P07564 | DENV2 CLGKSYAOMWTIMY FHRRDLRLAANAICSAVP SHWVPTSRTT WSIHAKHEWMTTEDMLAV P29991 | DENV2 CIGKSYDOMWSIMY FHRRDLRLAANAICSAVPSHWVPT SRTTWSIHAKHEWMT TEDMLTV P29990 I DENV2 CLGKSYAQMWSLMYFHRRDLRLAANAICSAVPSHWVPTSRTTWSIHAKHEWMTTEDMLTV P14340 | DENV2 CLGKSYAQMWSLMYFHRRDLRLAANAICSAVPSHWVPTSRTTWSIHAKHEWMTTEDMLTV
P14337 | DENV2 CLGKSYAQMWSLMY FHRRDLRLAANAICSAVPSHWVPTSRTTWSIHAKHEWMTTEDMLTV P27909 | DENV1 CLGKSYAQMWQLMY FHRRDLRLAANAICSAVPVDWVPTSRTTWSIHAHHOWMTTEDMLSY P17763 | DENV1 CLGKSYAQMWQLMY FHRRDLRLAANAICSAVPVDWVPTSRTTWSIHAHHOWMTTEDML,SV 5 Q5UB 1 | DENV 3 CLGKAYAOMWSLMY FHRRDLRLASNAIC SAVPVHWVPT SRTTWSIHAHHOWMTTEDMLTV Q6DLVO | DENV 3 CLGKAYAQMWSIMY EHRRDLRLASNAICSAVPVHWVTSRTTWS IHAHHOWMTTEDMLTV
VVPMVTQLAMTDTTPFGQQRVEKEKVDTRT PQPKPGTRVVMT TTANWLWALLGRKKNPRL 12 VIPMVTOLAMTDTTPFGQQRVEKEKVDTRT PQPKPGTRMVMT T TANWLWALLGKKKNPRL 12

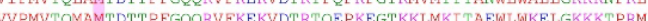

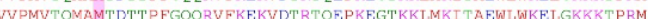

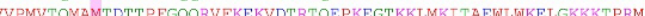
WVPMVTOVAMTDTTT PGQQRVVFKEKVDTRT OEPKEGTKKLMKTTA EWL WKEIGKKKTPRM 12 VVPMVTOMAMTDTTPFGQQRVFKEKVDTRTOEPKEGTKKLMKTTA AWLWKETGKKKTPRM 2 VL PTVTOVAMTDTT PEGOORVFKEKVDTRTOEPKEGTKKLMKTTAENTLIKETGKKKTPRM I PMYT OTAMTDTTP FGQORVFKEKVDTRT PRAKRGTAOTMEVTAKWLWGETSRNKKPRI I I MMVTOTAMTDTT'PFGQQRVFKEKVDTRT PKAKRGTAOTMEVTARWL,WGFLSRNKRPPI 12 VVPMVTQMAMTDTTPEGQQRVEKEKVDTRT PRPMPGTRKVMEITAEWLWRTLGRNKRPRI 2 VVPMVTOMAMTDTTPFGQQRVEKEKVDTRTPRPLPGTRKVMGITAEWLWRTLGRNKRPRL 120 343

MMGKREKKLGEFGRAKGSRAIWYMWLGARFLEFEALGFLNEDHWFGRENSWSGVEGEGLH 240 MMGKREKKLGEFGRAKGSRAIWYMWLGARFLEFEALGFLNEDHWFGRENSWSGVEGEGLH 240 MMGKREKKLGEFGRARGSRAIWYMWLGARFLEFEALGFLNEDHWFGRENSWSGVEGEGLH 2 MYGRREKLGEFGKAKGSRAIWYMWLGARFLEEEALGELNEDHWFSRENSLSGVEGEGLH IMGKREKKLGEFGKAKGSRAIWYMWLGARFLEFEALGFLNEDHWESRENSLSGVEGEGLH IMGKREKKLGEFGKAKGSRAIWYMWLGARELEEEALGELNEDHWFSRENSLSGVEGEGLH MMGKREKKLGEFGKAKGSRAIWYMWLGARFLEFEALGFLNEDHWFSRENSLSGVEGEGLH MMGKREKKLGEFGKAKGSRAIWYMWLGARFLEFEALGFLNEDHWESRENSLSGVEGEGLH MMGKREKKLGEFGKAKGSRAIWYMWLGARFLEFEALGFMNEDHWESRENSLSGVEGEGLH MMGKREKKLGEFGKAKGSRAIWYMWLGARFLEFEALGFMNEDHWFSRENSLSGVEGEGLH \begin{tabular}{l} 
MMGKREKKLGE FGKAKGSRAIWYMWLGARYLEE EALGFLNEDHWFSRENSYSGVEGEGLH \\
MMGKREKKLGEFGKAKGSRAIWYMWLGVRYLEFEALGFLNEDHWFSRENSYSGVEGEGLH \\
\hline
\end{tabular}

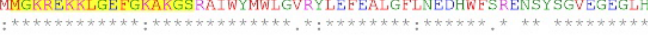

QNKVVKVLRPTPKGAVMDI I SRKDQRGSGQVGTYGL NT FTNMEVQLIRQMEAEGVITRDD 360 QNKVVKVLRPTPRGAVMDI I SRKDQRGSGQVGTYGLNT FTNMEVQLIRQMEAEGVITQDD
ONKVVKVLRPTPKGAVMDI ISRKDQRGSGOVGTYGLNT FTNMEVOLIRQMEAEGVITODD QNKVWVIRPTPKGAVMDI I SRKDQRGSGQVGT YGLNT FTNMEVQLIRQMEAEGVI TQDD
QNKVVRVRPTPRGTVMDIISRRDQRGSGQVGTYGLNT FTNMEAQLIRQMEGEGI FKSIQ

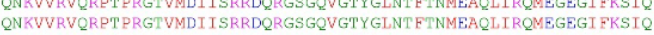
NKVVRVQRPT PRGTVMDI ISRRDQRGSGQVGTYGLNT FTNMEAQLIRQMEGEGVFKSIQ 36 W

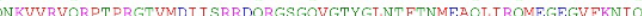
QNKVVRVQRPAKNGTVMDVISRRDQRGSGQVGTYGLNTETNMEVOLIROMESEGI PFPSE ONKVVPVORPAKNGTVMDVISRRDORGSGOVGTYGL NT ETNMEAOLIROMESEGT FSPSE QNKVVRVQRPAKNGTVMDVISRRDQRGSGQVGTYGLNT FTNMEAQLIRQMES EGIFSPSE
QNKVVKVQRPT PKGTVMDIISRKDQRGSGQVGTYGNT FTNMEAQLVRQMEGEGVLSKAD QNKVVKVQRPTPTGTVMDI I SRKDQRGSGQVGTYGLNT FTNMEAQLVRQMEGEGVLTKAD 36

QWEPSKGWKNWQEVPECSHHFHKI FMKDGRS IVVPCRNQDELIGRARISQGAGWSLRETA 48 QWEPSKGWKNWQEVP FCSHHFHKI FMKDGRSSIVPCRNQDELIGRARISQGAGWSLRETA 480 QWEPSKGWKNWQEVP ECSHHFHKI FMKDGRSIVVPCRNQDELIGRARISQGAGWSLRETA 49 QWEPSRGWNDWTQVPFCSHHFHEL IMKDGRTLVVPCRNQDELIGRARISQGAGWSLRETA OWEPSRGWNDWTQVPECSHHFHELVMKDGRVLVVPCRNQDELI GRARISQGAGWSLKETA QNEPSRGWNDWTQVP ECSHHFHELIMKDGRVLVVPCRNQDELI GRARISQGAGWSLRETA CWEPSRGWIDTTOUPFCSHEFELIMRGRVIVVPCRNODELIGRARISOGAGWSLRETA QWEPSRGWNDWTQVPFCSHHFHELIMKDGRVLVVPCRNQDELIGRARI SQGAGWSLRETA OWEPSKGWNDWQQVP FCSHHFHOLIMKDGREIVVPCRNQDELVGRARVSQGAGWSLRETA QWEPSKGWNDWQQVP FCSHHFHOLIMKDGREIVVPCRNQDELVGRARVSQGAGWSLRETA
OWOPSKGWHDWOQVP ECSHHEHELIMKDGRKLVVPCRPQDELIGRARISOGAGWSLKETA QWQPSKGWHDWQQVPFCSHHFHELIMKDGRKLVVPCRPQDELIGRARI SQGAGWSLRETA 4 737

WNRVWIEDNPNMI DKTPVHSWEDIPYLGKREDLWCGSLIGLSSRATWAKNIOTAITOVRN 600 WNRVWIEDNPNMT DKT PVVHSWEDI PYLGKREDLWCGSLIGLSSRATWAKNI HTAITQVRN WNRVWIEDNPNMTDKTPVHSWEDI PYLGKREDLWCGSLIGLSSRATWAKNI HTAITQVRN WNKVWILENPWMEDKTPVESWEEIPYLGKREDQWCGSLIGLTSRATWAKNIQTAINQVRS WNRVWIOENPWMEDKTPVESWEEVPYLGKREDQWCGSLIGLTSRATWAKNIQTAINQVRS WNRVWIQENPWMEDKTPVESWEEI PYLGKREDQWCGSLIGIT TRATWAKNI QAAINOVRS WNRVWIOENPWMEDKTPVESWEEI PYLGKREDQWCGSLIGLTSRATWAKNI QAAINQVRS WNRVWIQEN PWMEDKT PVESWEEI PYLGKREDQWCGSLI GLT SRATWAKNI QAAINQVRS 作

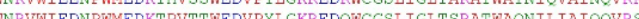
WNRVWIEENPWMEDKT PVTTWENVPYLGKREDQWCGSLI GITSRATWAQNI PTAIQQVRS

Q2YHFO IDENV4 LIGKEEYVDYMPVMKRYSAHEESEGVLP09866 | DENV4 LIGKEEYVDYMPVMKRYSAPSESEGV Q58HT7 | DENV4 LIGKEEYVDYMPVMKRYYSAPEESEGVLPO7564IDENV2 ITGMEYTDYMPSMKRFRREEEAGGVH P07564 DENV2 LIGNEEYTDYMPSMKRERREEEEAGVLM P29991 | DENV2 LIGNEEYTDYMPSMKRFRREEEEAGVLW P14340 IDENV2 LITNEEYTDYMPSMKRFRKEEEEAGVL P14337 | DENV2 LIGNEEYTDYMPSMKRFRREEEEEAGVLW P27909|DENV1 LIGNENYLDYMTSMKRFKNESDPEGALM P17763 | DENV1 LIGNENYLDEMTSMKREKNESDPEGGAL Q5UB51 I DENV3 LIGDEEELDYMPSMKRERKEEESEGAIW Q6DUVOLENV ITGNEELDYMPSMKRERKEEESEGAIW

\begin{tabular}{|c|c|c|c|c|c|c|c|c|c|c|c|c|c|c|}
\hline & & Q2YHFO & P09866 & Q58HT7 & P12823 & P07564 & P29991 & P29990 & P14340 & P14337 & P27909 & P17763 & Q5UB51 & Q6DLVO \\
\hline 1: & Q2YHEOIDENV4 & 100.00 & 97.61 & 98.72 & 76.52 & 75.72 & 75.40 & 75.88 & 76.52 & 76.04 & 75.24 & 75.08 & 78.95 & 78.63 \\
\hline 2: & P09866| DENV4 & 97.61 & 100.00 & 98.88 & 77.00 & 75.88 & 75.56 & 76.04 & 76.68 & 76.36 & 75.40 & 75.24 & 78.47 & 78.31 \\
\hline 3: & Q58HT7 | DENV4 & 98.72 & 98.88 & 100.00 & 76.52 & 75.72 & 75.40 & 75.88 & 76.52 & 76.20 & 75.08 & 74.92 & 78.63 & 78.31 \\
\hline 4: & P12823|DENV2 & 76.52 & 77.00 & 76.52 & 100.00 & 96.81 & 96.33 & 96.81 & 97.45 & 96.97 & 80.19 & 80.03 & 80.38 & 80.54 \\
\hline 5: & P07564|DENV2 & 75.72 & 75.88 & 75.72 & 96.81 & 100.00 & 97.13 & 97.61 & 98.41 & 97.93 & 80.35 & 80.19 & 80.22 & 80.06 \\
\hline & P29991 | DENV2 & 75.40 & 75.56 & 75.40 & 96.33 & 97.13 & 100.00 & 99.52 & 98.09 & 98.2 & 79.39 & 79.2 & 79.2 & 79.27 \\
\hline $7:$ & P29990| DENV2 & 75.88 & 76.04 & 75.88 & 96.81 & 97.61 & 99.52 & 100.00 & 98.56 & 98.72 & 79.55 & 79.39 & 79.74 & 79.74 \\
\hline 8: & P14340|DENV2 & 76.52 & 76.68 & 76.52 & 97.45 & 98.41 & 98.09 & 98.56 & 100.00 & 98.88 & 80.19 & 80.03 & 80.70 & 80.86 \\
\hline 9: & P14337| DENV2 & 76.04 & 76.36 & 76.20 & & & 98.25 & & 98.88 & 100.00 & & 79. & .06 & 80.06 \\
\hline 10: & P27909/DENV1 & 75.2 & 75.40 & 75.08 & 8 & 80.35 & 79.39 & 79.55 & 80.19 & 80.03 & 100.00 & 98.09 & 1.02 & 81.34 \\
\hline & P17763|DENV 1 & 75.08 & 75.24 & 74.92 & 80.03 & 80.19 & 79.23 & 79.39 & 80.03 & 79.87 & 98.09 & 100.00 & 80.70 & 80.70 \\
\hline & Q5UB51| DENV 3 & 78.95 & 78.47 & 78.63 & 80.38 & 80.22 & 79.27 & 79.74 & 80.70 & 80.06 & 81.02 & $\begin{array}{r}80.70 \\
\end{array}$ & 100.00 & 97.61 \\
\hline 13 & Q6DLVO I DENV3 & 78.63 & 78.31 & 78.31 & 80.54 & 80.06 & 79.27 & 79.74 & 80.86 & 80.06 & 81.34 & 80.70 & 97.61 & 100.00 \\
\hline
\end{tabular}

Figure I Multiple sequence alignment of NS5 RNA-dependent RNA polymerases of the different dengue virus serotypes (above) and the corresponding percent identitity matrix (below).

Notes: Pink boxes indicate the aminoacid position of M343, T4I3, and R737 for DENV-3 considering the full length of NS5 (MTase N-terminal + RdRp C-terminal) or the equivalent position for the other serotypes. Yellow boxes indicate missing residues in the crystallographic data. Orange boxes indicate the GDD motif. For each sequence, the UniProt code for the full polyprotein of the virus | SEROTYPE is indicated.

Abbreviation: NS5, nonstructural protein 5. 
capacity of computational calculation, molecular docking is performed considering a flexible ligand and a rigid receptor. The aim of molecular docking is to predict the structure of the ligand-receptor complex using computational methods. AutoDock/Vina incorporates Monte Carlo simulated annealing, evolutionary, genetic, and Lamarckian genetic algorithm methods to model the ligand flexibility while keeping the receptor rigid. ${ }^{35}$ Also, AutoDock Vina uses in its scoring function the AMBER force field, which computes the terms of the contributions of van der Waals interactions, hydrogen bonding, electrostatic interactions, conformational entropy, and desolvation. ${ }^{36}$ In the current study, 3D models of NS5 RpRd of the four serotypes of DENV were docked with two sets of compounds: molecules sharing $\geq 70 \%$ structural similarity with NITD-1, NITD-2, and NITD-2921 and 37,840 compounds from the SuperNatural $\mathrm{II}^{23}$ database with optimum pharmacokinetic and toxicological profiles.
Tables 2 and 3 show the free energy variation $(\Delta G)$ calculated using AutoDocK/Vina for the best docking scores, obtained for the NITD-related compounds and the SuperNatural II database compounds, respectively. In addition, hydrogen bonds and direct contacts based on van der Waals radii for compounds with the lowest free energy variations $(\Delta \mathrm{G} \leq-10.5 \mathrm{kcal} / \mathrm{mol})$ are available at http://docking. umh.es/. The calculated $\mathrm{K}_{\mathrm{D}}\left(\mathrm{K}_{\mathrm{D}}=\exp ^{\Delta \mathrm{G} / \mathrm{RT}}\right)$ for compounds with $\Delta \mathrm{G} \leq-10.5 \mathrm{kcal} / \mathrm{mol}$ is in the nanomolar or subnanomolar range that was used as a threshold to filter the docking results. ${ }^{28}$ Therefore, $\Delta \mathrm{G}$ values represent the first filter in the selection of putative inhibitors among the compounds studied. Additional filters were applied before proposing the final candidates. The free energy variation is a representative value of the number and intensity of the atomic interactions between the receptor (protein) and the ligand, and can thus be considered a baseline comparison for the selection of lead compounds

Table 2 Molecular docking analysis for NITD-related and potential inhibitor compounds (candidate molecules in this study) at the binding site of NS5 RdRp located in the RNA template tunnel

\begin{tabular}{|c|c|c|c|c|c|c|c|}
\hline $\begin{array}{l}\text { Ligand } \\
\text { name }\end{array}$ & $\begin{array}{l}\Delta \mathbf{G}(\mathrm{kcal} / \mathrm{mol}) \\
\text { mean } \pm \mathbf{S D}\end{array}$ & $\begin{array}{l}\text { Ligand } \\
\text { name }\end{array}$ & $\begin{array}{l}\Delta \mathbf{G}(\mathbf{k c a l} / \mathrm{mol}) \\
\text { mean } \pm \mathbf{S D}\end{array}$ & $\begin{array}{l}\text { Ligand } \\
\text { name }\end{array}$ & $\begin{array}{l}\Delta \mathbf{G}(\mathbf{k c a l} / \mathrm{mol}) \\
\text { mean } \pm \mathbf{S D}\end{array}$ & $\begin{array}{l}\text { Ligand } \\
\text { name }\end{array}$ & $\begin{array}{l}\Delta \mathbf{G}(\mathrm{kcal} / \mathrm{mol}) \\
\text { mean } \pm \mathbf{S D}\end{array}$ \\
\hline \multicolumn{2}{|c|}{ NS5 RdRp of DENV-I } & \multicolumn{2}{|c|}{ NS5 RdRp of DENV-2 } & & & & \\
\hline NITD-I & $-8.6 \pm 0.5$ & NITD-I & $-8.5 \pm 0.6$ & 11999940 & $-11.2 \pm 0.9$ & 11612450 & $-11.0 \pm 0.7$ \\
\hline NITD-2 & $-9.6 \pm 0.5$ & NITD-2 & $-9.9 \pm 0.8$ & 71815510 & $-11.2 \pm 0.6$ & 22867347 & $-11.0 \pm 1.0$ \\
\hline NITD-29 & $-10.1 \pm 0.8$ & NITD-29 & $-10.8 \pm 1.2$ & 53019114 & $-11.2 \pm 1.3$ & 53834657 & $-11.0 \pm 0.7$ \\
\hline 91166410 & $-11.5 \pm 1.2$ & 53019095 & $-11.8 \pm 1.2$ & 68563659 & $-11.2 \pm 0.9$ & 86608192 & $-11.0 \pm 1.1$ \\
\hline 88881717 & $-11.5 \pm 1.1$ & 53019000 & $-11.7 \pm 1.6$ & 90644640 & $-11.2 \pm 0.8$ & 58550574 & $-11.0 \pm 0.6$ \\
\hline 53019095 & $-11.4 \pm 1.0$ & 58847065 & $-11.6 \pm 0.6$ & $74937 \mid 25$ & $-11.2 \pm 1.0$ & 87254969 & $-11.0 \pm 1.0$ \\
\hline 58509828 & $-11.4 \pm 1.3$ & 58847008 & $-11.6 \pm 0.6$ & 5330687 & $-11.2 \pm 1.0$ & 91982228 & $-11.0 \pm 0.8$ \\
\hline 86592137 & $-11.4 \pm 0.7$ & 59558635 & $-11.5 \pm 0.6$ & 53019157 & $-I I . I \pm I .0$ & 58117326 & $-11.0 \pm 1.0$ \\
\hline 87254969 & $-11.4 \pm 0.7$ & 53019136 & $-11.5 \pm 1.7$ & 53019118 & $-\mid I . I \pm I .1$ & 86592137 & $-11.0 \pm 1.0$ \\
\hline 58847008 & $-11.4 \pm 0.6$ & 91166410 & $-11.5 \pm 1.3$ & 57483735 & $-11.1 \pm 0.9$ & 46228828 & $-11.0 \pm 0.8$ \\
\hline 24320265 & $-11.4 \pm 0.8$ & 53019046 & $-11.5 \pm 1.2$ & 42623761 & $-\mid I . I \pm I . I$ & 57590479 & $-11.0 \pm 0.8$ \\
\hline 5330687 & $-11.3 \pm 1.0$ & 58509828 & $-11.5 \pm 1.3$ & 58847188 & $-11.1 \pm 0.6$ & 24818217 & $-11.0 \pm 1.1$ \\
\hline 71815510 & $-11.3 \pm 0.6$ & $57883 \mid 47$ & $-11.4 \pm 0.8$ & 11612953 & $-I I . I \pm I .0$ & 58716236 & $-11.0 \pm 0.9$ \\
\hline 53019000 & $-11.3 \pm 1.0$ & 88881717 & $-11.4 \pm 1.2$ & 59120485 & $-11.1 \pm 0.9$ & 53019023 & $-11.0 \pm 1.4$ \\
\hline $58847 \mid 88$ & $-11.3 \pm 0.3$ & 76724063 & $-11.4 \pm 0.8$ & 71815570 & $-11.1 \pm 0.7$ & 99173129 & $-11.0 \pm 1.0$ \\
\hline 58847065 & $-11.3 \pm 0.7$ & 4982894 & $-\mid I .4 \pm 1.1$ & 60118013 & $-11.1 \pm 0.9$ & 16749692 & $-11.0 \pm 0.6$ \\
\hline 46228732 & $-11.3 \pm 0.9$ & 90644642 & $-11.4 \pm 1.0$ & 46228732 & $-11.1 \pm 0.9$ & 53019026 & $-11.0 \pm 1.5$ \\
\hline 4982894 & $-11.2 \pm 1.1$ & I52375I & $-11.3 \pm 1.1$ & 90644637 & $-11.1 \pm 1.0$ & 10163912 & $-11.0 \pm 0.9$ \\
\hline 58550574 & $-11.2 \pm 0.7$ & 60138427 & $-11.3 \pm 0.9$ & 53098093 & $-11.1 \pm 0.9$ & 53019135 & $-11.0 \pm 1.6$ \\
\hline 71815377 & $-11.2 \pm 0.5$ & 21114517 & $-11.3 \pm 0.9$ & 99437562 & $-11.1 \pm 1.2$ & II 503697 & $-11.0 \pm 1.1$ \\
\hline 16749628 & $-11.2 \pm 0.5$ & 60118830 & $-11.3 \pm 0.9$ & 68490845 & $-\mid I . I \pm 0.8$ & 58117469 & $-11.0 \pm 0.9$ \\
\hline 53019136 & $-11.2 \pm 0.9$ & 53019143 & $-11.3 \pm 1.3$ & 17240799 & $-11.1 \pm 0.9$ & 67017052 & $-11.0 \pm 1.1$ \\
\hline 66966190 & $-11.1 \pm 0.3$ & 11591299 & $-11.3 \pm 1.0$ & 10116388 & $-I I . I \pm 0.9$ & 83275648 & $-11.0 \pm 1.3$ \\
\hline 53019046 & $-11.1 \pm 0.9$ & 46926790 & $-11.2 \pm 0.8$ & 60402568 & $-I I . I \pm I .0$ & $2159834 \mid$ & $-11.0 \pm 1.0$ \\
\hline 66661763 & $-\mid I .1 \pm 0.6$ & 67023200 & $-11.2 \pm 0.8$ & 59508095 & $-11.1 \pm 1.2$ & 44419986 & $-11.0 \pm 0.9$ \\
\hline 57955372 & $-11.1 \pm 0.3$ & 44605230 & $-11.2 \pm 1.4$ & 51038723 & $-\mid I . I \pm I . I$ & 58518208 & $-11.0 \pm 0.4$ \\
\hline 16749692 & $-11.1 \pm 0.6$ & $6025008 I$ & $-11.2 \pm 1.1$ & 69707773 & $-11.0 \pm 0.9$ & II547552 & $-11.0 \pm 0.8$ \\
\hline $7|8| 544 \mid$ & $-I I . I \pm 0.5$ & 59558530 & $-11.2 \pm 0.6$ & 57955382 & $-11.0 \pm 0.6$ & 59558378 & $-11.0 \pm 0.9$ \\
\hline 58044822 & $-\mid I .1 \pm 0.8$ & 66662216 & $-11.2 \pm 0.7$ & 22556722 & $-11.0 \pm 1.0$ & 59508029 & $-11.0 \pm 1.1$ \\
\hline
\end{tabular}

(Continued) 
Table 2 (Continued)

\begin{tabular}{|c|c|c|c|c|c|c|c|}
\hline $\begin{array}{l}\text { Ligand } \\
\text { name }\end{array}$ & $\begin{array}{l}\Delta \mathbf{G}(\mathrm{kcal} / \mathrm{mol}) \\
\text { mean } \pm \mathbf{S D}\end{array}$ & $\begin{array}{l}\text { Ligand } \\
\text { name }\end{array}$ & $\begin{array}{l}\Delta \mathbf{G}(\mathrm{kcal} / \mathrm{mol}) \\
\text { mean } \pm \mathbf{S D}\end{array}$ & $\begin{array}{l}\text { Ligand } \\
\text { name }\end{array}$ & $\begin{array}{l}\Delta \mathbf{G}(\mathrm{kcal} / \mathrm{mol}) \\
\text { mean } \pm \mathbf{S D}\end{array}$ & $\begin{array}{l}\text { Ligand } \\
\text { name }\end{array}$ & $\begin{array}{l}\Delta \mathbf{G}(\mathrm{kcal} / \mathrm{mol}) \\
\text { mean } \pm \mathbf{S D}\end{array}$ \\
\hline \multicolumn{2}{|c|}{ NS5 RdRp of DENV-I } & \multicolumn{2}{|c|}{ NS5 RdRp of DENV-2 } & & & & \\
\hline $2159834 \mid$ & $-11.0 \pm 1.2$ & 24672975 & $-11.2 \pm 0.6$ & 22587546 & $-11.0 \pm 0.9$ & 53019073 & $-|I .0 \pm I|$. \\
\hline 22556747 & $-11.0 \pm 1.0$ & 59069323 & $-11.2 \pm 1.2$ & 58044822 & $-11.0 \pm 0.6$ & 53310399 & $-11.0 \pm 1.0$ \\
\hline 42598694 & $-11.0 \pm 0.5$ & 9804722 & $-11.2 \pm 1.2$ & 10239163 & $-11.0 \pm 0.7$ & 86608190 & $-11.0 \pm 1.0$ \\
\hline 71815570 & $-11.0 \pm 0.6$ & 5330694 & $-11.2 \pm 1.1$ & $5608544 I$ & $-11.0 \pm 1.4$ & 59113200 & $-\mid I .0 \pm I . I$ \\
\hline 11591299 & $-11.0 \pm 0.7$ & 66661763 & $-11.2 \pm 0.7$ & 44068150 & $-11.0 \pm 1.2$ & 2307085 & $-11.0 \pm 0.9$ \\
\hline 90644637 & $-11.0 \pm 0.9$ & 88881725 & $-11.2 \pm 0.2$ & 2408779 & $-11.0 \pm 0.8$ & 58847051 & $-11.0 \pm 0.6$ \\
\hline 53239274 & $-11.0 \pm 1.0$ & 71815377 & $-11.2 \pm 0.4$ & 60117771 & $-11.0 \pm 1.0$ & & \\
\hline 90644642 & $-11.0 \pm 1.0$ & 88884325 & $-11.2 \pm 0.4$ & 58094574 & $-11.0 \pm 0.9$ & & \\
\hline \multicolumn{8}{|c|}{ NS5 RdRp of DENV-4 } \\
\hline NITD-I & $-8.9 \pm 0.3$ & 22556722 & $-11.3 \pm 1.3$ & 5330659 & $-\mid I . I \pm I . I$ & 56244330 & $-11.0 \pm 1.0$ \\
\hline NITD-2 & $-10.2 \pm 0.5$ & 58187960 & $-11.3 \pm 0.7$ & 53041346 & $-11.1 \pm 1.0$ & 4498761 & $-11.0 \pm 0.9$ \\
\hline NITD-29 & $-10.8 \pm 0.7$ & 71016043 & $-11.3 \pm 0.6$ & 58117268 & $-11.1 \pm 0.6$ & 6129755 & $-11.0 \pm 0.8$ \\
\hline 4982894 & $-12.2 \pm 1.4$ & 58117308 & $-11.3 \pm 0.5$ & 66605204 & $-\mid I . I \pm 0.7$ & 10410064 & $-11.0 \pm 0.7$ \\
\hline 2408779 & $-12.0 \pm 1.1$ & 59121600 & $-11.3 \pm 0.8$ & 25065563 & $-11.1 \pm 0.9$ & 23627602 & $-11.0 \pm 0.8$ \\
\hline 59558635 & $-11.9 \pm 1.0$ & 6524590 & $-11.2 \pm 1.1$ & 53884638 & $-11.1 \pm 1.3$ & 26283419 & $-11.0 \pm 0.9$ \\
\hline 44605230 & $-11.9 \pm 0.5$ & 55797299 & $-11.2 \pm 0.8$ & 57483798 & $-11.1 \pm 0.8$ & 45167069 & $-11.0 \pm 1.2$ \\
\hline 71815510 & $-11.8 \pm 0.8$ & II52554| & $-11.2 \pm 1.2$ & 9930411 & $-11.1 \pm 1.3$ & 51925674 & $-11.0 \pm 0.6$ \\
\hline 59558530 & $-11.8 \pm 0.6$ & 76810150 & $-11.2 \pm 1.8$ & 25138236 & $-1 \mathrm{I} . \mathrm{I} \pm 0.7$ & 5579027I & $-11.0 \pm 1.0$ \\
\hline $8888|7| 7$ & $-11.8 \pm 1.3$ & $5608544 I$ & $-11.2 \pm 0.9$ & 25154812 & $-11.1 \pm 0.9$ & 57467031 & $-11.0 \pm 1.0$ \\
\hline 91166410 & $-11.8 \pm 1.3$ & 68698259 & $-11.2 \pm 1.0$ & 58117332 & $-11 . I \pm 0.4$ & 59558574 & $-11.0 \pm 0.8$ \\
\hline 58509828 & $-11.7 \pm 1.2$ & 99173125 & $-11.2 \pm 0.7$ & 59962443 & $-11.1 \pm 0.6$ & 60118484 & $-11.0 \pm 0.4$ \\
\hline 16749692 & $-11.6 \pm 1.1$ & II9998।3 & $-11.2 \pm 0.9$ & 77152204 & $-11.1 \pm 0.7$ & 66662021 & $-\mid 1.0 \pm 1.1$ \\
\hline 46228732 & $-11.6 \pm 1.0$ & 25138230 & $-11.2 \pm 0.8$ & 99501210 & $-11.1 \pm 1.3$ & 69707178 & $-11.0 \pm 0.7$ \\
\hline 68701918 & $-11.6 \pm 1.2$ & 53098094 & $-11.2 \pm 0.3$ & 16749694 & $-11.1 \pm 1.0$ & 22063077 & $-11.0 \pm 0.6$ \\
\hline 60402568 & $-11.6 \pm 0.9$ & 59875861 & $-11.2 \pm 1.1$ & 19288807 & $-11.1 \pm 1.0$ & 56022032 & $-11.0 \pm 0.4$ \\
\hline 25065772 & $-11.6 \pm 1.0$ & 68496294 & $-11.2 \pm 1.3$ & 46926790 & $-|1| \pm 1.2$. & 59558479 & $-11.0 \pm 0.9$ \\
\hline 68702576 & $-11.6 \pm 1.1$ & 60205770 & $-11.2 \pm 0.9$ & 58787557 & $-11.1 \pm 1.3$ & 66662543 & $-11.0 \pm 0.6$ \\
\hline 66661763 & $-11.6 \pm 1.0$ & 21114517 & $-11.2 \pm 0.6$ & 76541785 & $-\mid 1 . I \pm 0.7$ & 4498763 & $-11.0 \pm 0.8$ \\
\hline 58094574 & $-11.6 \pm 0.9$ & 25065362 & $-11.2 \pm 1.0$ & 20765416 & $-11.1 \pm 1.0$ & 10392307 & $-11.0 \pm 0.9$ \\
\hline 76724063 & $-11.6 \pm 1.4$ & 54127944 & $-11.2 \pm 0.8$ & 25066204 & $-\mid I . I \pm I . I$ & 46377852 & $-11.0 \pm 0.7$ \\
\hline 66662686 & $-11.6 \pm 0.7$ & 44303726 & $-11.2 \pm 1.0$ & 27563621 & $-\mid I . I \pm I . I$ & 5084867I & $-11.0 \pm 0.9$ \\
\hline 57955382 & $-11.5 \pm 1.3$ & 53098093 & $-11.2 \pm 0.4$ & 46602120 & $-\mid I . I \pm I . I$ & 59212388 & $-11.0 \pm 0.9$ \\
\hline 88884325 & $-11.5 \pm 1.8$ & 56925033 & $-11.2 \pm 1.9$ & 53098102 & $-11.1 \pm 0.5$ & 66604901 & $-11.0 \pm 0.8$ \\
\hline $57883 \mid 47$ & $-11.5 \pm 1.4$ & 66606315 & $-11.2 \pm 1.0$ & 16431302 & $-11.1 \pm 1.0$ & 68894072 & $-\mid 1.0 \pm 1.1$ \\
\hline 6025008 I & $-11.5 \pm 0.8$ & 86608190 & $-11.2 \pm 1.2$ & 3554749 & $-11.1 \pm 0.8$ & 89886799 & $-11.0 \pm 0.9$ \\
\hline 11591299 & $-11.5 \pm 1.1$ & 89361165 & $-11.2 \pm 0.6$ & 25174057 & $-11.1 \pm 1.0$ & 44605022 & $-11.0 \pm 0.7$ \\
\hline 17240799 & $-11.5 \pm 0.6$ & 89361072 & $-11.2 \pm 0.6$ & 58509782 & $-11.1 \pm 1.3$ & 60402552 & $-11.0 \pm 0.9$ \\
\hline 25065775 & $-11.5 \pm 1.0$ & 20765428 & $-11.2 \pm 1.0$ & 69144161 & $-11.1 \pm 0.9$ & 76808085 & $-11.0 \pm 1.2$ \\
\hline $44 \mid 76508$ & $-11.5 \pm 1.2$ & 58044822 & $-11.2 \pm 0.8$ & 23627601 & $-I I . I \pm I . I$ & $173597 \mid 8$ & $-11.0 \pm 0.6$ \\
\hline 46885243 & $-11.5 \pm 0.9$ & 59239811 & $-11.2 \pm 1.0$ & 69579276 & $-11.1 \pm 0.9$ & 35767226 & $-\mid 1.0 \pm 1.1$ \\
\hline $15605 \mid 85$ & $-11.5 \pm 0.6$ & 146429 & $-11.2 \pm 1.3$ & 10116388 & $-11.0 \pm 0.9$ & 58768495 & $-11.0 \pm 0.9$ \\
\hline 5330687 & $-11.5 \pm 1.3$ & 11612450 & $-11.2 \pm 1.0$ & 25065360 & $-11.0 \pm 1.0$ & 4710567 & $-11.0 \pm 0.7$ \\
\hline 25065774 & $-11.5 \pm 1.0$ & $44068 I 50$ & $-11.2 \pm 0.9$ & 76310566 & $-11.0 \pm 0.8$ & 9799922 & $-\mid 1.0 \pm 1.2$ \\
\hline 66662216 & $-11.5 \pm 1 . \mid$ & 53098204 & $-11.2 \pm 0.4$ & 46833812 & $-11.0 \pm 1.8$ & 11756868 & $-11.0 \pm 1.0$ \\
\hline 60118013 & $-11.5 \pm 1.1$ & 25065776 & $-11.2 \pm 0.9$ & 10206429 & $-11.0 \pm 0.8$ & 16589824 & $-\mid 1.0 \pm 1.0$ \\
\hline $8888 \mid 725$ & $-11.5 \pm 1.1$ & 53310399 & $-11.2 \pm 0.8$ & 30870527 & $-11.0 \pm 0.8$ & 44155098 & $-11.0 \pm 0.7$ \\
\hline 53019000 & $-11.4 \pm 1.0$ & 18710168 & $-11.2 \pm 0.7$ & 44580357 & $-11.0 \pm 1.1$ & 44159652 & $-11.0 \pm 0.7$ \\
\hline 60118830 & $-\mid I .4 \pm 0.5$ & 50848824 & $-11.2 \pm 1.0$ & 59304633 & $-11.0 \pm 0.5$ & 57483735 & $-11.0 \pm 0.7$ \\
\hline 11612953 & $-11.4 \pm 0.9$ & 98939207 & $-11.2 \pm 0.6$ & $6669488 I$ & $-11.0 \pm 0.9$ & 59121601 & $-11.0 \pm 1.0$ \\
\hline 86592137 & $-11.4 \pm 0.4$ & 59375041 & $-11.2 \pm 1.8$ & 9537955 & $-11.0 \pm 1.6$ & $58|1732|$ & $-11.0 \pm 0.4$ \\
\hline 87254969 & $-11.4 \pm 0.4$ & 60494790 & $-11.2 \pm 0.6$ & 10069263 & $-11.0 \pm 0.8$ & 9886055 & $-11.0 \pm 1.2$ \\
\hline
\end{tabular}


Table 2 (Continued)

\begin{tabular}{|c|c|c|c|c|c|c|c|}
\hline $\begin{array}{l}\text { Ligand } \\
\text { name }\end{array}$ & $\begin{array}{l}\Delta \mathbf{G}(\mathrm{kcal} / \mathrm{mol}) \\
\text { mean } \pm \mathrm{SD}\end{array}$ & $\begin{array}{l}\text { Ligand } \\
\text { name }\end{array}$ & $\begin{array}{l}\Delta \mathbf{G}(\mathrm{kcal} / \mathrm{mol}) \\
\text { mean } \pm \mathrm{SD}\end{array}$ & $\begin{array}{l}\text { Ligand } \\
\text { name }\end{array}$ & $\begin{array}{l}\Delta \mathbf{G}(\mathrm{kcal} / \mathrm{mol}) \\
\text { mean } \pm \mathbf{S D}\end{array}$ & $\begin{array}{l}\text { Ligand } \\
\text { name }\end{array}$ & $\begin{array}{l}\Delta \mathbf{G}(\mathrm{kcal} / \mathrm{mol}) \\
\text { mean } \pm \mathbf{S D}\end{array}$ \\
\hline \multicolumn{8}{|c|}{ NS5 RdRp of DENV-4 } \\
\hline $6011777 \mid$ & $-11.4 \pm 0.4$ & 5330699 & $-11.2 \pm 1.4$ & 41273686 & $-\mid 1.0 \pm 1.1$ & 52886452 & $-11.0 \pm 0.5$ \\
\hline 25138229 & $-11.4 \pm 0.8$ & 25065773 & $-11.2 \pm 0.8$ & 46228828 & $-11.0 \pm 0.9$ & 66604312 & $-11.0 \pm 0.9$ \\
\hline 46228865 & $-11.4 \pm 0.7$ & 56057109 & $-11.2 \pm 0.6$ & 57483762 & $-11.0 \pm 0.4$ & $687 \mid 4559$ & $-|1.0 \pm 1|$. \\
\hline 10342132 & $-I I .4 \pm 1.0$ & 58550574 & $-11.2 \pm 0.9$ & 68701232 & $-11.0 \pm 1.0$ & 6129075 & $-11.0 \pm 0.6$ \\
\hline 67133415 & $-11.4 \pm 1.4$ & 10228015 & $-11.2 \pm 0.9$ & 53018993 & $-11.0 \pm 0.5$ & | | & $-11.0 \pm 0.6$ \\
\hline 71815570 & $-11.4 \pm 0.6$ & 39906246 & $-11.2 \pm 0.5$ & 58069426 & $-11.0 \pm 1.0$ & 16065158 & $-11.0 \pm 1.2$ \\
\hline |667534| & $-I I .4 \pm I .0$ & 58064300 & $-11.2 \pm 1.2$ & 11760627 & $-11.0 \pm 1.1$ & 18710165 & $-11.0 \pm 0.7$ \\
\hline 24320265 & $-11.4 \pm 1.0$ & 60402580 & $-11.2 \pm 1.1$ & 16583630 & $-11.0 \pm 0.6$ & 19665288 & $-11.0 \pm 1.0$ \\
\hline 68695702 & $-11.4 \pm 1.2$ & 68698167 & $-11.2 \pm 0.9$ & 20637893 & $-11.0 \pm 1.0$ & 22556747 & $-11.0 \pm 1.3$ \\
\hline 50848923 & $-11.4 \pm 0.9$ & 20765438 & $-11.2 \pm 1.1$ & 22909360 & $-11.0 \pm 0.9$ & $245 \mid 2675$ & $-11.0 \pm 0.8$ \\
\hline 87254968 & $-\mid 1.4 \pm 1.3$ & 51038723 & $-11.2 \pm 0.5$ & $5748376 I$ & $-11.0 \pm 0.4$ & 25138237 & $-11.0 \pm 0.9$ \\
\hline 16749628 & $-|I .4 \pm I|$. & $6048 \mid 456$ & $-11.2 \pm 0.8$ & 58607039 & $-\mid 1.0 \pm 1.1$ & 57483734 & $-11.0 \pm 0.5$ \\
\hline 25066203 & $-11.4 \pm 0.9$ & 56085277 & $-11.2 \pm 1.2$ & 59615666 & $-11.0 \pm 0.9$ & 57878492 & $-11.0 \pm 1.0$ \\
\hline 86608192 & $-11.4 \pm 1.3$ & 56025347 & $-11.1 \pm 1.3$ & 59986516 & $-11.0 \pm 0.9$ & 88042824 & $-11.0 \pm 0.8$ \\
\hline $36245 I$ & $-11.3 \pm 1.3$ & II 999940 & $-1 \mathrm{I} .1 \pm 0.7$ & $6048 \mid 455$ & $-11.0 \pm 0.8$ & 90299314 & $-11.0 \pm 0.9$ \\
\hline 10477276 & $-11.3 \pm 0.9$ & 53019143 & $-11.1 \pm 0.9$ & 66599533 & $-11.0 \pm 0.6$ & 5330694 & $-\mid 1.0 \pm 1.4$ \\
\hline 71815377 & $-11.3 \pm 0.5$ & 58117326 & $-11.1 \pm 0.2$ & 69138857 & $-11.0 \pm 0.4$ & 45856807 & $-11.0 \pm 0.6$ \\
\hline 59375107 & $-11.3 \pm 1.9$ & 67023384 & $-11.1 \pm 0.7$ & 76541767 & $-11.0 \pm 0.9$ & 2647295 & $-11.0 \pm 0.8$ \\
\hline 67023200 & $-11.3 \pm 0.7$ & 44251670 & $-11.1 \pm 1.0$ & 89856954 & $-\mid 1.0 \pm 1.4$ & 6197109 & $-11.0 \pm 0.7$ \\
\hline 68490845 & $-11.3 \pm 0.9$ & $44|78| 68$ & $-11.1 \pm 1.0$ & $1000228 \mid$ & $-11.0 \pm 0.8$ & 9804722 & $-11.0 \pm 1.3$ \\
\hline $444 \mid 9986$ & $-11.3 \pm 0.8$ & 53019026 & $-\mid I . I \pm I . I$ & $4262376 I$ & $-11.0 \pm 0.9$ & 20637894 & $-11.0 \pm 0.7$ \\
\hline $5280 \mid 43$ & $-11.3 \pm 0.8$ & $58064 \mid 37$ & $-\mid I . I \pm I . I$ & 51035546 & $-11.0 \pm 0.6$ & 21378092 & $-11.0 \pm 0.6$ \\
\hline 57878498 & $-11.3 \pm 0.8$ & 16327670 & $-\mid 1.1 \pm 0.8$ & 51035547 & $-11.0 \pm 0.4$ & 25070620 & $-\mid 1.0 \pm 1.5$ \\
\hline 18710166 & $-11.3 \pm 1.2$ & 24548686 & $-11.1 \pm 0.9$ & 56214202 & $-\mid 1.0 \pm 1.1$ & 31895387 & $-11.0 \pm 0.8$ \\
\hline 59880286 & $-11.3 \pm 1.2$ & 86608206 & $-\mid I . I \pm 1.3$ & 59558378 & $-11.0 \pm 1.0$ & 54347717 & $-11.0 \pm 1.3$ \\
\hline 59144489 & $-11.3 \pm 1.2$ & 68700123 & $-11.1 \pm 1.2$ & 71149319 & $-11.0 \pm 0.6$ & 55760732 & $-11.0 \pm 1.0$ \\
\hline 18710167 & $-11.3 \pm 1.5$ & 46885245 & $-11.1 \pm 1.0$ & 25065560 & $-|1.0 \pm 1|$. & 57955372 & $-11.0 \pm 1.0$ \\
\hline 60402567 & $-11.3 \pm 0.9$ & 53019136 & $-11.1 \pm 0.9$ & 58117279 & $-11.0 \pm 0.6$ & $58975|3|$ & $-11.0 \pm 0.5$ \\
\hline $7|8| 544 \mid$ & $-11.3 \pm 0.6$ & 60621794 & $-\mid 1.1 \pm 0.8$ & 90731410 & $-11.0 \pm 1.0$ & 59121588 & $-|1.0 \pm 1|$. \\
\hline 25065987 & $-11.3 \pm 1.2$ & 68559862 & $-\mid 1.1 \pm 1.0$ & 102119533 & $-\mid 1.0 \pm 1.1$ & 59558337 & $-11.0 \pm 0.8$ \\
\hline 11317629 & $-11.3 \pm 1.0$ & 85874649 & $-\mid I . I \pm 1.0$ & 44605023 & $-11.0 \pm 0.7$ & 59880283 & $-11.0 \pm 1.2$ \\
\hline 44419982 & $-11.3 \pm 0.9$ & 5330697 & $-\mid I . I \pm 1.4$ & 46231533 & $-11.0 \pm 0.6$ & 66605319 & $-11.0 \pm 0.7$ \\
\hline 47037799 & $-11.3 \pm 0.7$ & 16376567 & $-\mid I . I \pm 0.8$ & 54087042 & $-11.0 \pm 0.5$ & 66661799 & $-11.0 \pm 0.9$ \\
\hline 50848762 & $-11.3 \pm 0.8$ & 53019095 & $-11.1 \pm 0.9$ & 5739I50I & $-11.0 \pm 0.5$ & 74937104 & $-11.0 \pm 0.5$ \\
\hline 53019046 & $-11.3 \pm 0.9$ & 20765417 & $-11.1 \pm 1.2$ & 59973115 & $-11.0 \pm 1.4$ & & \\
\hline 58518208 & $-11.3 \pm 1.8$ & 22556882 & $-11.1 \pm 1.2$ & 69707773 & $-11.0 \pm 0.9$ & & \\
\hline 89856935 & $-11.3 \pm 1.2$ & 99455350 & $-11.1 \pm 0.9$ & 59212517 & $-11.0 \pm 0.6$ & & \\
\hline 10239163 & $-11.3 \pm 1.1$ & 2947808 & $-11.1 \pm 0.8$ & 41927843 & $-11.0 \pm 0.7$ & & \\
\hline
\end{tabular}

Notes: The name of each ligand was obtained from the PubChem database (https://pubchem.ncbi.nlm.nih.gov/). ${ }^{22}$ The name of the compounds NITD-I, -2 , and -29 was taken from Niyomrattanakit et al. ${ }^{21}$ The table shows the estimated binding free energy variation ${ }^{31}$ (mean \pm SD). The maps of the interacting residues of the protein with the best ligands and $\Delta \mathrm{G}$ values among the candidate compounds are available at http://docking.umh.es/

Abbreviations: NS5, nonstructural protein 5; RdRp, RNA-dependent RNA polymerase; SD, standard deviation.

in the process of drug design. ${ }^{28}$ Several compounds have $\Delta \mathrm{G}$ values in the range of -10.5 to $-10.0 \mathrm{kcal} / \mathrm{mol}$ (see full list at http://docking.umh.es/). Interestingly, the calculated $\Delta \mathrm{G}$ values for all of the tested compounds against $3 \mathrm{D}$ models of DENV-3 NS5 RdRp are greater than $-10 \mathrm{kcal} / \mathrm{mol}$. Calculated $\Delta \mathrm{G}$ values for NITD-1, -2 , and -29 against all DENV serotypes are worse than those of the selected compounds in Tables 2 and 3. On the other hand, we must remember that these compounds are often inactive in cell culture. ${ }^{21}$ Thus, additional filters were applied on these selected compounds (Tables 2 and 3) to further characterize them in terms of pharmacokinetics and toxicity.

\section{Prediction of ADMET profiles}

In addition to its efficiency to inhibit the target protein, other factors such as absorption, biodistribution, rate at 
Table 3 Molecular docking analysis for natural compounds

\begin{tabular}{|c|c|c|c|c|c|}
\hline \multicolumn{2}{|c|}{ RdRp of DENV-I } & \multicolumn{2}{|c|}{ RdRp of DENV-2 } & \multicolumn{2}{|c|}{ RdRp of DENV-4 } \\
\hline $\begin{array}{l}\text { Ligand } \\
\text { name }\end{array}$ & $\begin{array}{l}\Delta \mathbf{G}(\mathrm{kcal} / \mathrm{mol}) \\
\text { mean } \pm \mathbf{S D}\end{array}$ & $\begin{array}{l}\text { Ligand } \\
\text { name }\end{array}$ & $\begin{array}{l}\Delta \mathbf{G}(\mathrm{kcal} / \mathrm{mol}) \\
\text { mean } \pm \mathbf{S D}\end{array}$ & $\begin{array}{l}\text { Ligand } \\
\text { name }\end{array}$ & $\begin{array}{l}\Delta \mathbf{G}(\mathrm{kcal} / \mathrm{mol}) \\
\text { mean } \pm \mathrm{SD}\end{array}$ \\
\hline SN00091933 & $-10.8 \pm 0.7$ & SN00I5I425 & $-11.1 \pm 0.8$ & SN000I6053 & $-11.2 \pm 1.0$ \\
\hline SN0007409I & $-10.7 \pm 0.7$ & SN000I0280 & $-10.9 \pm 0.8$ & SN00057073 & $-I I . I \pm I . I$ \\
\hline SN0009I667 & $-10.7 \pm 0.8$ & SN00023794 & $-10.9 \pm 0.7$ & SN00063622 & $-11.1 \pm 1.2$ \\
\hline SN00306679 & $-10.6 \pm 1.0$ & SN00372243 & $-10.9 \pm 0.9$ & SN00058424 & $-I I . I \pm I . I$ \\
\hline SN00I5I425 & $-10.6 \pm 0.9$ & SN00II 5885 & $-10.8 \pm 1.1$ & SN00058828 & $-\mid 1.1 \pm 1.1$ \\
\hline SN00366028 & $-10.5 \pm 1.0$ & SN000I8927 & $-10.8 \pm 0.5$ & SN00057669 & $-11.1 \pm 1.1$ \\
\hline SN00372243 & $-10.5 \pm 1.0$ & SN000I6053 & $-10.8 \pm 0.5$ & SN0023047I & $-11.1 \pm 1.3$ \\
\hline SN00074088 & $-10.5 \pm 0.9$ & SN00057073 & $-10.7 \pm 1.1$ & SN00057220 & $-11.0 \pm 1.2$ \\
\hline SN000I0280 & $-10.5 \pm 0.6$ & SN00081933 & $-10.6 \pm 1.1$ & SN0005706I & $-11.0 \pm 1.2$ \\
\hline SN00I 27203 & $-10.5 \pm 1.1$ & SN00I 27203 & $-10.6 \pm 1.3$ & SN0030356I & $-11.0 \pm 1.2$ \\
\hline SN000I6053 & $-10.5 \pm 0.6$ & SN00058424 & $-10.6 \pm 1.1$ & SN00058587 & $-10.9 \pm 1.1$ \\
\hline SN0023047I & $-10.5 \pm 0.9$ & SN00063622 & $-10.6 \pm 1.0$ & SN00366028 & $-10.9 \pm 0.8$ \\
\hline \multirow[t]{21}{*}{ SN0024500I } & $-10.5 \pm 0.7$ & SN00379696 & $-10.6 \pm 0.6$ & SN00I 27203 & $-10.8 \pm 0.8$ \\
\hline & & SN00396968 & $-10.6 \pm 0.8$ & SN00303525 & $-10.8 \pm 1.0$ \\
\hline & & SN000I 4343 & $-10.6 \pm 0.9$ & SN000I6042 & $-10.7 \pm 1.4$ \\
\hline & & SN00057669 & $-10.5 \pm 1.0$ & SN000I0280 & $-10.7 \pm 0.6$ \\
\hline & & SN00282274 & $-10.5 \pm 0.9$ & SN00IIII882 & $-10.6 \pm 1.0$ \\
\hline & & SN0038I633 & $-10.5 \pm 1.2$ & SN00282274 & $-10.6 \pm 1.0$ \\
\hline & & SN00004095 & $-10.5 \pm 0.6$ & SN003I7979 & $-10.6 \pm 1.0$ \\
\hline & & SN0024500I & $-10.5 \pm 0.4$ & SN000I8896 & $-10.6 \pm 0.9$ \\
\hline & & SN00058828 & $-10.5 \pm 1.0$ & SN000264I4 & $-10.6 \pm 1.2$ \\
\hline & & SN00397486 & $-10.5 \pm 0.9$ & SN00I5I956 & $-10.6 \pm 0.6$ \\
\hline & & SN00282224 & $-10.5 \pm 0.9$ & SN00282224 & $-10.6 \pm 1.2$ \\
\hline & & SN00009726 & $-10.5 \pm 0.9$ & SN00004I46 & $-10.6 \pm 0.9$ \\
\hline & & & & SN00I5I425 & $-10.6 \pm 1.1$ \\
\hline & & & & SN00379696 & $-10.6 \pm 1.0$ \\
\hline & & & & SN000I 2056 & $-10.5 \pm 0.7$ \\
\hline & & & & SN0038I633 & $-10.5 \pm 0.7$ \\
\hline & & & & SN00II 5885 & $-10.5 \pm 0.8$ \\
\hline & & & & SN00I48919 & $-10.5 \pm 0.6$ \\
\hline & & & & SN00276030 & $-10.5 \pm 0.8$ \\
\hline & & & & SN00249I37 & $-10.5 \pm 0.7$ \\
\hline & & & & SN00091933 & $-10.5 \pm 0.9$ \\
\hline
\end{tabular}

Notes: The maps of the interacting residues of the protein with the best ligands and $\Delta \mathrm{G}$ values among the candidate compounds are available at http://docking.umh.es/. The name of each ligand was obtained from the SuperNatural database (http://bioinf-applied.charite.de/supernatural_new/index.php?site=home). The table shows estimated binding free energy variation ${ }^{31}$ (mean $\pm S D$ ) of potential inhibitors at the binding site of NS5 RdRp located in the RNA template tunnel.

Abbreviations: NS5, nonstructural protein 5; RdRp, RNA-dependent RNA polymerase; SD, standard deviation.

which it is metabolized, excretion, and toxicity (ie, ADMET profile) upon its administration contributes to the overall success of a drug candidate. ${ }^{37}$ Moreover, it is recognized that employing computational ADMET, in combination with in vivo and in vitro predictions in early stage in the drug discovery process, helps to reduce the number of potential safety problems ${ }^{38}$ In the next selection step, different paramaters were calculated to predict which compounds from Tables 2 and 3 would exhibit optimal ADMET profiles. The logarithm of partition coefficient between $n$-octanol and water is a well-established measure of the compound's hydrophilicity. It has been shown that for compounds to have a reasonable probability of being well absorbed, their calculated $\log \mathrm{P}$ value must not be greater than $5.0 .^{33}$ Low solubility of a compound negatively impacts its absorption and, consequently, its biodistribution. Therefore, compounds with a calculated $\log S$ (logarithm base 10 of the solubility measured in mol/L) less than -4.0 were discarded. ${ }^{33}$ Compounds with fragment-based druglikeness $\leq 0$ and a drugscore (calculated as a combination of druglikeness, calculated $\log \mathrm{P}$, calculated $\log \mathrm{S}, \mathrm{MW}$, and toxicity risks $) \leq 0.5$ were also discarded. Most orally administered drugs have an MW of $\leq 500$, a calculated $\log \mathrm{P} \leq 5$, five or fewer hydrogen bond donor sites, and ten or fewer hydrogen bond acceptor 
sites (Lipinski's rule of five). ${ }^{32}$ Molecules violating more than one of these rules were ruled out. Also, mutagenicity, tumorigenicity, irritancy, and reproductive effects calculated with DataWarrior software ${ }^{33}$ were used to further identify compounds with low tendency for toxicity. Table 4 shows the compounds which satisfied all of the criteria described above, which reduced the number of putative NS5 RdRp inhibitors to 39 compounds, which is $0.010 \%$ of all compounds in the two databases screened in this study. Figure 2 shows the calculated $\Delta \mathrm{G}$ and molecular formula for the 39 selected compounds against each of the four serotypes of DENV. The calculated $\Delta \mathrm{G}$ for the selected compounds is

Table 4 Physicochemical parameters for selected compounds based on molecular docking analysis

\begin{tabular}{|c|c|c|c|c|c|c|c|c|c|c|}
\hline Compound & $\%$ ABS & $\begin{array}{l}\text { TPSA } \\
\left(A^{2}\right)\end{array}$ & MW & clogS & clogP & HBA & HBD & $\begin{array}{l}\text { Ro5 } \\
\text { violations }\end{array}$ & $\begin{array}{l}\text { Drug } \\
\text { score }\end{array}$ & $\begin{array}{l}\text { Drug } \\
\text { likeness }\end{array}$ \\
\hline NITD-I & 66.05 & 124.5 & 373.1 & -3.39 & 0.544 & 8 & 2 & 0 & 0.21153 & -0.2761 \\
\hline NITD-2 & 71.91 & 107.5 & 433.1 & -4.61 & $2.4 I$ & 7 & 2 & 0 & 0.608586 & 1.9599 \\
\hline NITD-29 & 56.01 & 153.6 & 656.2 & -8.22 & 2.602 & 10 & 3 & I & 0.11867 & -3.1308 \\
\hline SN00II 5885 & 70.36 & 112 & 478.5 & -2.872 & 4.763 & 8 & 2 & 0 & 0.571559 & 3.9196 \\
\hline 71815570 & 70.67 & III.I & 478.2 & -4.08 & 3.935 & 8 & 3 & 0 & 0.613687 & $2.144 \mid$ \\
\hline 89856954 & 70.67 & III.I & 478.2 & -4.08 & 4.146 & 8 & 3 & 0 & 0.613687 & $2.144 \mid$ \\
\hline SN000I8896 & 71.60 & 108.4 & 487.6 & -3.608 & -0.06 & 8 & 3 & 0 & 0.682805 & 3.8613 \\
\hline SN00303525 & 71.60 & 108.4 & 482.5 & -3.791 & 0.732 & 8 & I & 0 & 0.542329 & $0.364 I$ \\
\hline SN00379696 & 72.57 & 105.6 & 443.5 & -0.855 & 2.249 & 9 & 2 & 0 & 0.800132 & 7.1765 \\
\hline SN00282274 & 72.78 & 105 & 418.4 & -1.829 & 1.623 & 9 & I & 0 & 0.725687 & 1.0679 \\
\hline SN00372243 & 72.78 & 105 & 432.4 & -1.938 & 1.921 & 9 & I & 0 & 0.707052 & 1.0873 \\
\hline SN00396968 & 72.78 & 105 & 418.4 & -1.829 & 1.623 & 9 & I & 0 & 0.725687 & 1.0679 \\
\hline SN0005706I & 73.19 & 103.8 & 463.5 & -3.33 & -0.003 & 7 & 0 & 0 & 0.736422 & 5.5881 \\
\hline SN00057220 & 73.19 & 103.8 & 493.9 & -3.876 & 0.942 & 7 & 0 & 0 & 0.660014 & 6.9371 \\
\hline SN00057669 & 73.19 & 103.8 & 477.5 & -3.454 & 0.437 & 7 & 0 & 0 & 0.709263 & 5.2853 \\
\hline SN00058587 & 73.19 & 103.8 & 479.9 & -3.752 & 0.502 & 7 & 0 & 0 & 0.688934 & 7.2363 \\
\hline SN00081933 & 73.19 & 103.8 & 473.5 & -3.689 & 1.987 & 7 & 0 & 0 & 0.68905 I & 12.562 \\
\hline SN00276030 & 73.36 & 103.3 & 453.5 & -3.312 & 2.68 & 6 & 4 & 0 & 0.642133 & 1.2805 \\
\hline SN00I5I425 & 73.50 & 102.9 & 483.5 & -2.059 & 2.768 & 8 & 2 & 0 & 0.579584 & 0.4603 \\
\hline SN00004I 46 & 74.91 & 98.8 & 476.9 & -3.894 & I.44| & 8 & 2 & 0 & 0.660046 & 2.9203 \\
\hline SN00009726 & 75.47 & 97.2 & 465.5 & -2.537 & 4.503 & 8 & I & 0 & 0.587977 & 2.0354 \\
\hline SN00306679 & 75.78 & 96.3 & 413.4 & $-1.49 \mid$ & 1.835 & 8 & 2 & 0 & 0.838983 & 7.1078 \\
\hline 50848824 & 76.57 & 94 & 489.2 & -3.75 & 0.864 & 8 & 0 & 0 & 0.63805 I & 8.2672 \\
\hline 55760732 & 76.57 & 94 & 475.2 & -2.95 & 0.661 & 8 & 0 & 0 & 0.710746 & 9.1186 \\
\hline SN00282224 & 76.74 & 93.5 & 402.4 & $-2.05 I$ & 2.283 & 8 & I & 0 & 0.815395 & 3.1154 \\
\hline SN00397486 & 76.74 & 93.5 & 402.4 & -2.051 & 2.283 & 8 & I & 0 & 0.815395 & 3.1154 \\
\hline 5084867| & 77.64 & 90.9 & 494.2 & -3.25 & 1.125 & 8 & 0 & 0 & 0.533224 & 4.7277 \\
\hline 57590479 & 77.95 & 90 & 486.2 & -3.49 & 2.117 & 8 & I & 0 & 0.675188 & 3.0453 \\
\hline SN000I8927 & 79.26 & 86.2 & 496.6 & -3.01 & -2.63 & 9 & 4 & 0 & 0.708905 & 6.3156 \\
\hline SN00023794 & 79.26 & 86.2 & 494.6 & -3.28 & -0.002 & 8 & 3 & 0 & 0.697577 & 6.3156 \\
\hline SN00III 882 & 79.43 & 85.7 & 458.5 & -3.072 & 4.922 & 8 & I & 0 & 0.569557 & 3.6342 \\
\hline 39906246 & 80.81 & 81.7 & 464.1 & -3.46 & 1.655 & 7 & 0 & 0 & 0.693327 & 5.794 \\
\hline SN000I 2056 & 81.37 & 80.1 & 477.6 & -2.58 & 4.976 & 7 & I & 0 & 0.563559 & 4.8245 \\
\hline SN003I 7979 & 81.47 & 79.8 & 491.5 & -2.988 & 3.963 & 8 & 0 & 0 & 0.605967 & 2.8022 \\
\hline SN000I6042 & 81.57 & 79.5 & 473.5 & -2.96 & 4.68 & 7 & 2 & 0 & 0.569749 & 2.744 \\
\hline SN00074088 & 81.64 & 79.3 & $4 \mid 8.4$ & -3.072 & $3.24 I$ & 7 & I & 0 & 0.69883 & 1.8624 \\
\hline SN0007409I & 81.64 & 79.3 & 418.4 & -3.072 & 3.241 & 7 & I & 0 & 0.69883 & 1.8624 \\
\hline SN000I 6053 & 82.81 & 75.9 & 485.6 & -3.12 & 3.398 & 7 & 2 & 0 & 0.629475 & 2.2823 \\
\hline SN000I4343 & 85.57 & 67.9 & 488.6 & -3.285 & 0.458 & 8 & 2 & 0 & 0.697442 & 3.8483 \\
\hline SN000264I 4 & 85.71 & 67.5 & 454.5 & -3.046 & 4.801 & 6 & I & 0 & 0.589066 & 4.2039 \\
\hline SN00366028 & 87.09 & 63.5 & 364.4 & -3.959 & 3.49 & 5 & I & 0 & 0.621615 & 0.9079 \\
\hline SN000I 0280 & 92.85 & 46.8 & 442.6 & -3.694 & 3.91 & 5 & I & 0 & 0.586606 & 1.4089 \\
\hline
\end{tabular}

Note: The NITD-I, -2, and -29 compounds, tested experimentally, ${ }^{21}$ are included for comparison.

Abbreviations: \% ABS, percentage of absorption; clogS, the estimated logarithm (base 10 ) of the solubility measured in mol/L; clogP, calculated logarithm of partition coefficient between n-octanol and water; HBA, number of hydrogen bond acceptors; HBD, number of hydrogen bond donors; MW, molecular weight; Ro5 violations, violation of Lipinski's rules; TPSA, topological polar surface area. 
A

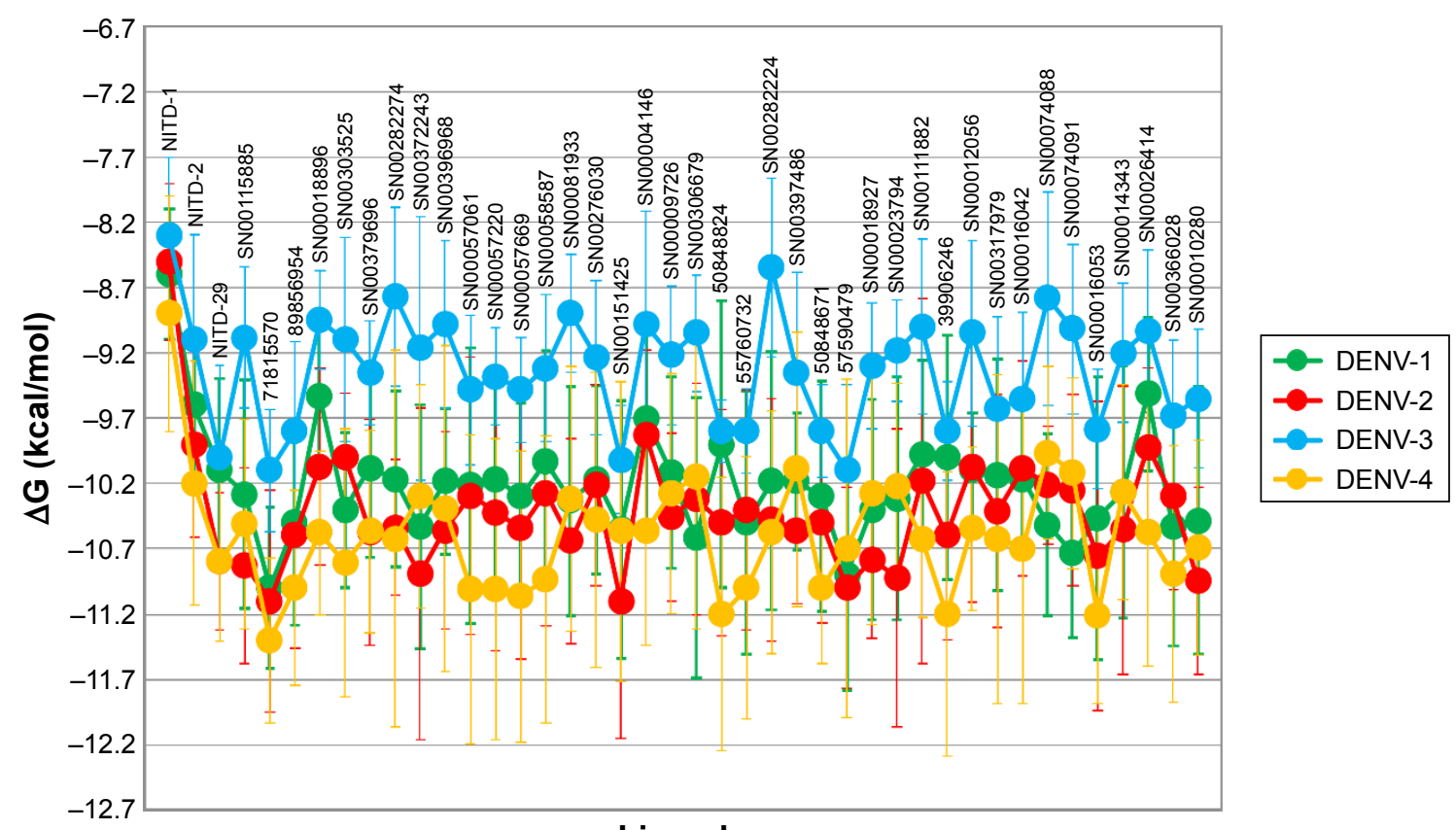

B

Ligands

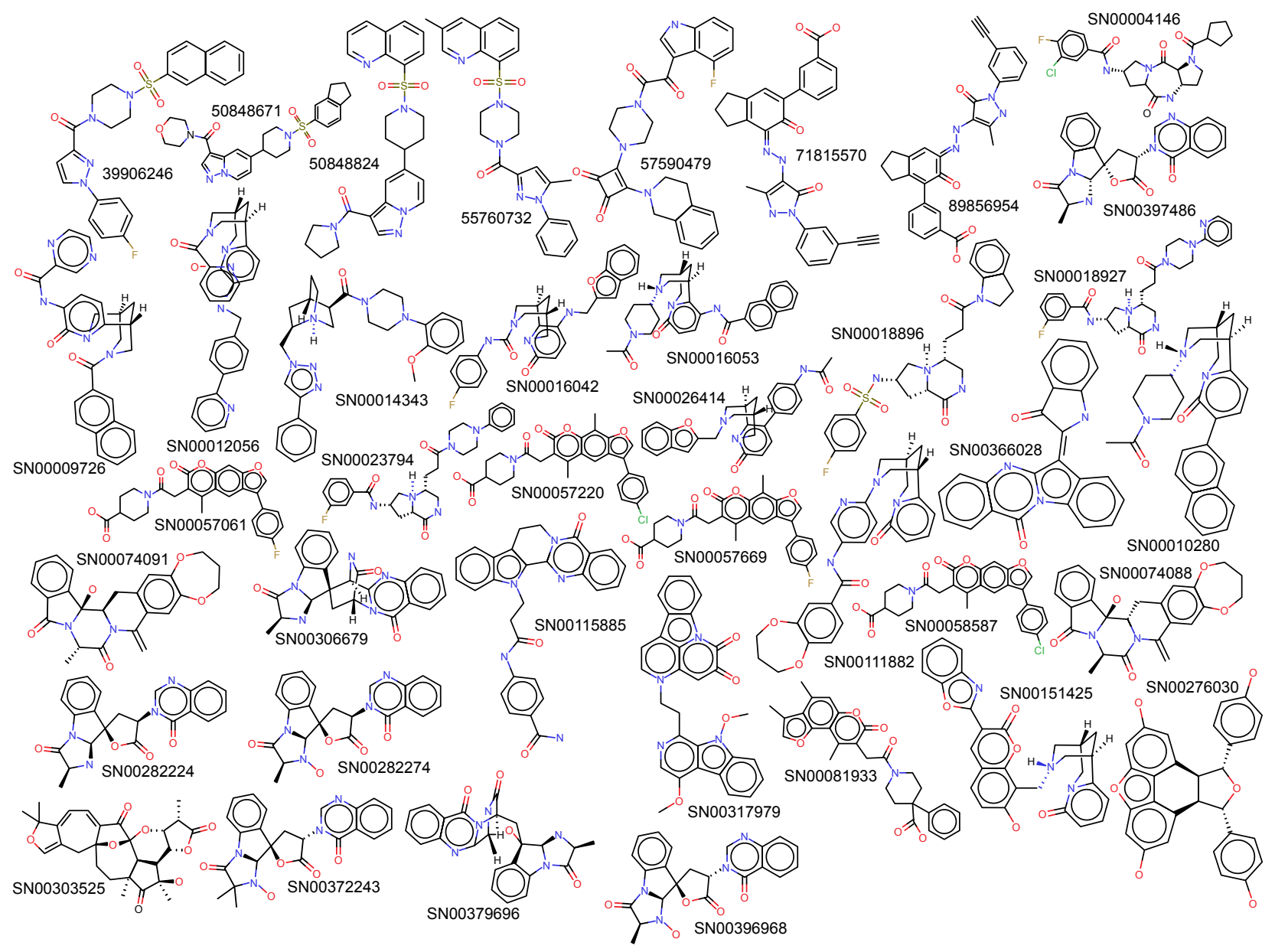

Figure 2 Comparison of the free energy variation $(\Delta G)$ for selected compounds based on molecular docking analysis against NS5 RdRps of all four serotypes of dengue virus (A). $\Delta G$ for NITD compounds is also included. (B) The molecular structures of the compounds represented in (A); the PubChem ID or the SuperNatural 2 ID is close to the structure of each compound.

Abbreviation: NS5, nonstructural protein 5. 
lower (ie, potentially better candidates) for DENV-2 and -4 and higher for DENV-3. Each compound interacts with multiple residues of the protein (Figure 3) after docking to the binding site of the RNA template tunnel. The interaction maps for the best compounds ( $\Delta \mathrm{G}<-10.5 \mathrm{kcal} / \mathrm{mol})$ were calculated and are available at http://docking.umh.es/. The TPSA has been correlated with passive molecular transport through membranes; therefore, TPSA is able to predict transport properties of drugs. Usually, compounds passively absorbed and with TPSA $<140 \AA^{2}$ are likely to exhibit high oral bioavailability. According to Zhao et al, ${ }^{39}$ the percentage of absorption can be estimated using the lineal equation: $\%$ of absorption $=109-0.345 \times$ TPSA. Using this formula, the calculated percentages of absorption for selected compounds ranged between $70.86 \%$ and $92.85 \%$ (Table 4 ). What is the behavior of NITD-1, -2, and -29 compounds in relation to the above parameters? None of these three compounds satisfied all of the criteria imposed in this study to select potential DENV NS5 RdRp inhibitors. NITD-1 and -29 have low percentages of absorption ( $66 \%$ and $56 \%$, respectively) (Table 4). Also, their calculated drug score value is less than 0.5 and their druglikeness is less than 0 (Table 4). The MW of NITD-29 is >500 Da, a violation of Lipinski's rule of five. NITD-2 presents a percentage of absorption of $72 \%$; however, its $\log \mathrm{S}$ value is less than -4 . The low aqueous solubility of drug candidates is a significant hurdle in many drug development projects.

We calculated additional pharmacokinetic properties following Cheng et $\mathrm{al}^{34}$ (Table 5). Almost all of the selected compounds showed optimum results for predicted human intestinal absorption and some compounds did so for transportation through blood-brain barrier and Caco-2. Also, the selected compounds showed no inhibitory side effects in terms of renal cation transport. The cytochrome P450 protein family catalyzes the oxidation of drugs and xenobiotics, generating more soluble compounds that are more readly removable from the body. ${ }^{40}$ These enzymes are expressed in the liver, small intestine (reducing drug bioavailability), lungs, placenta, and kidneys. Most of the selected putative inhibitors (Table 5) did not serve as substrates for cytochrome 2C9 and 2D6, whereas almost all of them were found to act as substrates for cytochrome P450 3A4. Inhibitors of cytochrome P450 decrease the enzymatic activity of these enzymes in a dose-dependent manner and promote the accumulation of drugs to toxic levels; therefore, it is desirable that the selected compounds do not serve as inhibitors of cytochrome P450.
Most of the compounds included in Table 5 satisfy this condition.

Today it is very useful to predict the risk of toxicity of drug candidates with bioinformatics tools. ${ }^{37}$ The toxicity risk predictor software identifies functional group similarity of the query molecule with the in vitro- and the in vivo-validated molecules included in software's built-in database; thus, similarity indicates a potential risk of toxicity. Table 6 presents the toxicity screening results for the selected compounds against DENV NS5 RdRp. None of the compounds presented potentially risks of tumorigenicity, mutagenicity, for reproductive function, or irritation. ${ }^{33}$ Similarly, the selected compounds were negative for AMES toxicity, did not potentially inhibit human ether-a-go-go-related genes, or exhibited no properties that posed significant toxicity risk for humans. The human ether-a-go-go-related gene channel is a voltage-gated potassium channel in cardiac cells, and is essential for cardiac repolarization. With the inhibition of this channel, the electrical depolarization and repolarization of the heart ventricles can be extended, leading to potentially fatal cardiac malfunction. ${ }^{41}$

\section{Conclusion}

The selected putative DENV NS5 RdRp inhibitors identified and characterized in this in silico study showed strong theoretical binding affinity (high negative free energy variation, $\Delta \mathrm{G})$, as determined by molecular docking against the binding site at the RNA template tunnel for the four DENV serotypes. This, to a great extent, is explained by the multiple sites of interaction of these compounds (Figure 3) with DENV NS5 RdRp. The selected compounds bind to the allosteric site located near the amino acids Met343, Arg737, and Thr413 in a similar way to NITD-29. ${ }^{21}$ Also, most of the selected compounds presented favorable druggability and optimum ADMET profiles, which suggests that as drug candidates, they will exhibit favourable traits, such as optimal absorption and biodistribution, compound stability, or low toxicity, all of which are critical for the success of a drug candidate. Further in vitro and in vivo studies will be required to confirm whether the in silico predicted ability of the selected compounds to inhibit DENV NS5 RdRp can be used to reduce DENV in live biological systems and strictly select those with the best potential to be used in real case scenarios. We believe that the information presented here will be useful for other laboratories interested in developing inhibitors against the NS5 RdRp of DENV. 

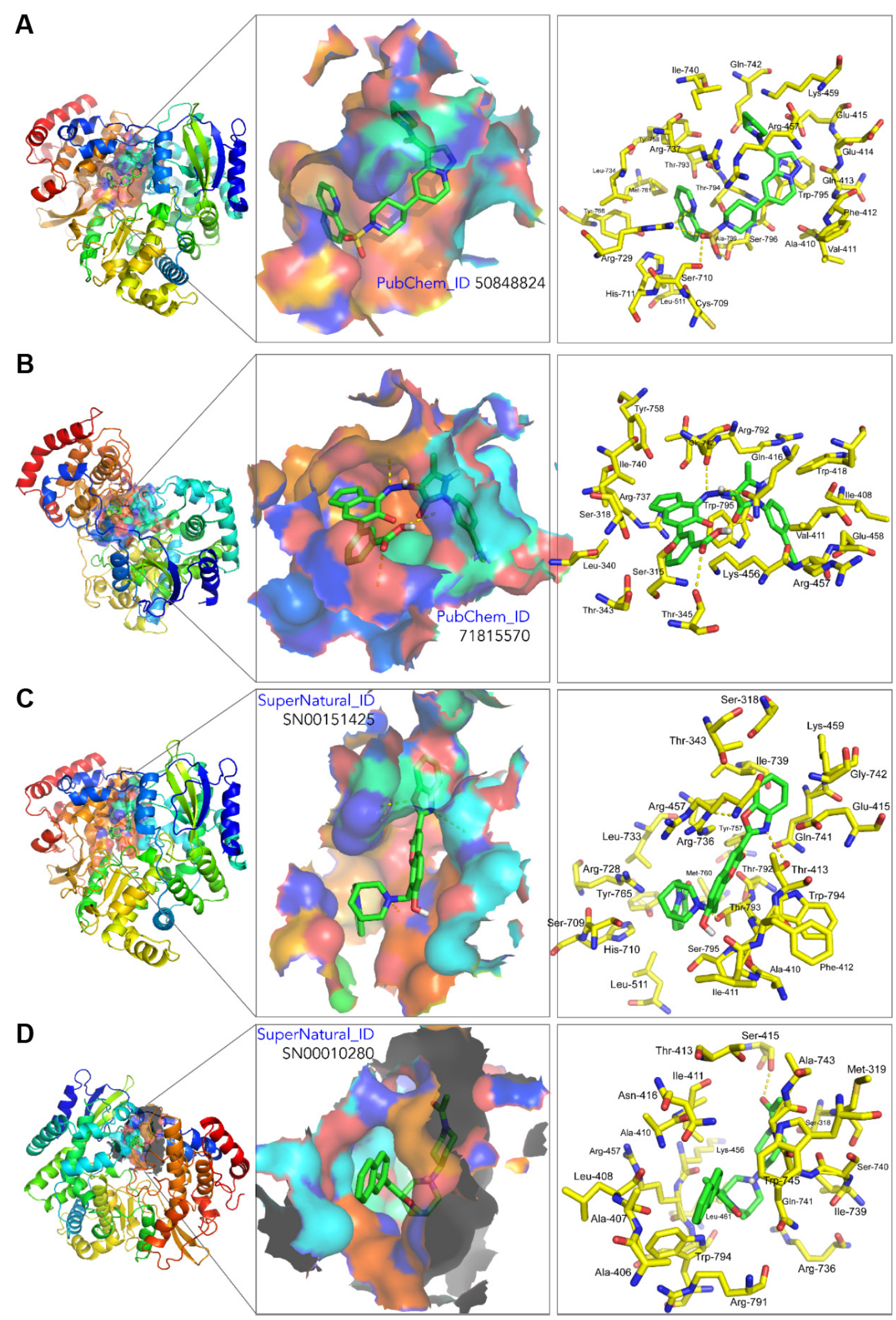

Figure 3 3D structure of the proteins NS5 RNA-dependent RNA polymerases of DENV-4 (A and B) and DENV-2 (C and D) showing the binding sites (left), the binding cavity (middle), and the main residues involved in the ligand-protein interaction of compound 50848824 (A), 71815570 (B), SN00151425 (C), and SN00010280 (D). Structure visualization was by PyMol I.8.2.0.

Abbreviations: 3D, three dimensional; NS5, nonstructural protein 5. 
Table 5 Predicted molecular pharmacokinetic properties of selected compounds against the NS5 RdRp

\begin{tabular}{|c|c|c|c|c|c|c|c|c|}
\hline \multirow[t]{2}{*}{ Compound } & \multicolumn{8}{|c|}{ ADME } \\
\hline & BBB & HIA & $\begin{array}{l}\text { Caco-2 } \\
\text { permeability }\end{array}$ & $\begin{array}{l}\text { Caco-2 permeability } \\
\text { (logPapp, cm/s) }\end{array}$ & $\begin{array}{l}\text { P-gp } \\
\text { substrate }\end{array}$ & $\begin{array}{l}\text { P-gp } \\
\text { inhibitor I }\end{array}$ & $\begin{array}{l}\text { P-gp } \\
\text { inhibitor II }\end{array}$ & $\begin{array}{l}\text { CYP450 2C9 } \\
\text { substrate }\end{array}$ \\
\hline NITD-I & BBB- & HIA+ & Caco-2- & 0.1784 & - & - & - & + \\
\hline NITD-2 & $\mathrm{BBB}+$ & HIA+ & Caco-2- & -0.0001 & - & - & + & - \\
\hline NITD-29 & $\mathrm{BBB}+$ & $\mathrm{HIA+}$ & Caco-2- & 0.2513 & - & - & + & + \\
\hline SN00II 5885 & $\mathrm{BBB}+$ & HIA+ & Caco-2- & 0.0438 & + & + & - & - \\
\hline 71815570 & BBB- & HIA+ & Caco-2- & 0.2006 & + & - & - & - \\
\hline 89856954 & BBB- & HIA+ & Caco-2- & 0.246 & + & - & - & - \\
\hline SN000I8896 & $\mathrm{BBB}+$ & HIA- & Caco-2- & 0.0127 & + & + & - & - \\
\hline SN00303525 & $\mathrm{BBB}+$ & $\mathrm{HIA}+$ & Caco-2- & 1.0792 & + & + & - & - \\
\hline SN00379696 & BBB- & $\mathrm{HIA}+$ & Caco-2- & 0.8915 & + & + & - & - \\
\hline SN00282274 & BBB- & $\mathrm{HIA}+$ & Caco-2- & 0.711 & + & - & - & - \\
\hline SN00372243 & BBB- & HIA+ & Caco-2- & 0.7707 & + & + & - & - \\
\hline SN00396968 & BBB- & HIA+ & Caco-2- & 0.711 & + & - & - & - \\
\hline SN0005706I & $\mathrm{BBB}+$ & HIA+ & Caco-2+ & 0.83 & + & - & - & - \\
\hline SN00057220 & $\mathrm{BBB}+$ & $\mathrm{HIA}+$ & Caco-2+ & 0.9218 & + & - & - & - \\
\hline SN00057669 & $\mathrm{BBB}+$ & HIA+ & Caco-2+ & 0.8885 & + & - & - & - \\
\hline SN00058587 & $\mathrm{BBB}+$ & HIA+ & Caco-2+ & 0.8622 & + & - & - & - \\
\hline SN00081933 & $\mathrm{BBB}+$ & HIA+ & Caco-2+ & 1.1446 & + & - & + & - \\
\hline SN00276030 & $\mathrm{BBB}+$ & HIA+ & Caco-2- & 0.8668 & - & - & - & - \\
\hline SN00I5I425 & BBB- & HIA+ & Caco-2- & 0.1968 & + & - & - & - \\
\hline SN00004I46 & $\mathrm{BBB}+$ & HIA+ & Caco-2- & 0.375 I & + & - & - & - \\
\hline SN00009726 & $\mathrm{BBB}+$ & HIA+ & Caco-2- & 0.7022 & + & + & - & - \\
\hline SN00306679 & $\mathrm{BBB}+$ & HIA+ & Caco-2- & 0.7746 & + & + & - & - \\
\hline 50848824 & $\mathrm{BBB}+$ & HIA+ & Caco-2- & 0.7676 & - & - & + & - \\
\hline 55760732 & $\mathrm{BBB}+$ & HIA+ & Caco-2- & 0.9103 & - & + & + & - \\
\hline SN00282224 & $\mathrm{BBB}+$ & HIA+ & Caco-2- & 0.7294 & + & + & - & - \\
\hline SN00397486 & $\mathrm{BBB}+$ & HIA+ & Caco-2- & 0.7294 & + & + & - & - \\
\hline 5084867| & $\mathrm{BBB}+$ & HIA+ & Caco-2- & 0.753 & - & + & - & - \\
\hline 57590479 & $\mathrm{BBB}+$ & HIA+ & Caco-2- & 0.5167 & + & + & + & - \\
\hline SN000I8927 & $\mathrm{BBB}+$ & HIA+ & Caco-2- & -0.2237 & + & + & - & - \\
\hline SN00023794 & $\mathrm{BBB}+$ & HIA+ & Caco-2- & 0.0423 & + & + & - & - \\
\hline SN00IIII882 & $\mathrm{BBB}+$ & HIA+ & Caco-2- & 0.7627 & + & + & - & - \\
\hline 39906246 & $\mathrm{BBB}+$ & HIA+ & Caco-2- & 0.7821 & - & + & + & - \\
\hline SN000I 2056 & $\mathrm{BBB}+$ & HIA+ & Caco-2- & 0.6186 & + & + & + & - \\
\hline SN003I 17979 & $\mathrm{BBB}+$ & HIA+ & Caco-2- & 0.823 & + & + & + & - \\
\hline SN000I6042 & $\mathrm{BBB}+$ & HIA+ & Caco-2- & 0.9035 & + & + & - & - \\
\hline SN00074088 & BBB- & $\mathrm{HIA}+$ & Caco-2+ & 1.175 & + & - & - & - \\
\hline SN0007409I & BBB- & HIA+ & Caco-2+ & 1.175 & + & - & - & - \\
\hline SN000I6053 & $\mathrm{BBB}+$ & HIA+ & Caco-2- & 0.4032 & + & + & - & - \\
\hline SN000I4343 & $\mathrm{BBB}+$ & HIA+ & Caco-2- & 0.6715 & + & + & + & - \\
\hline SN000264I4 & $\mathrm{BBB}+$ & HIA+ & Caco-2+ & 0.9295 & + & + & + & - \\
\hline SN00366028 & $\mathrm{BBB}+$ & HIA+ & Caco-2+ & 1.5359 & - & + & + & - \\
\hline SN000I0280 & $\mathrm{BBB}+$ & HIA+ & Caco-2- & 0.7316 & + & + & - & - \\
\hline
\end{tabular}

Notes: Compound names were obtained from PubChem ${ }^{22}$ and SuperNatural $11^{23}$ databases, respectively. ADME profiles of all compounds tested in this study are available at http://docking.umh.es/. All parameters have been calculated using the http://lmmd.ecust.edu.cn:8000/predict/site. ${ }^{34}$

Abbreviations: ADME, absorption, distribution, metabolism, and elimination; BBB, blood-brain barrier; CYP450, cytochrome P450; CYP IP, cytochrome P450 inhibitory promiscuity; HIA, human intestinal absorption; NS5, nonstructural protein 5; P-gp, P-glycoprotein; RdRp, RNA-dependent RNA polymerase; ROCT, renal organic cation transporter. 


\begin{tabular}{|c|c|c|c|c|c|c|c|c|}
\hline $\begin{array}{l}\text { CYP450 2D6 } \\
\text { substrate }\end{array}$ & $\begin{array}{l}\text { CYP450 3A4 } \\
\text { substrate }\end{array}$ & $\begin{array}{l}\text { CYP450 IA2 } \\
\text { inhibitor }\end{array}$ & $\begin{array}{l}\text { CYP450 2C9 } \\
\text { inhibitor }\end{array}$ & $\begin{array}{l}\text { CYP450 2D6 } \\
\text { inhibitor }\end{array}$ & $\begin{array}{l}\text { CYP450 2C19 } \\
\text { inhibitor }\end{array}$ & $\begin{array}{l}\text { CYP450 3A4 } \\
\text { inhibitor }\end{array}$ & CYP IP & ROCT \\
\hline- & - & - & - & - & - & - & Low & - \\
\hline - & - & - & + & - & - & - & Low & - \\
\hline - & - & - & + & - & - & + & High & - \\
\hline- & + & - & - & - & - & + & High & + \\
\hline- & + & - & + & - & - & + & High & - \\
\hline- & + & - & + & - & - & + & High & - \\
\hline- & + & - & - & - & - & - & High & - \\
\hline- & + & - & - & - & - & - & Low & - \\
\hline - & + & - & - & - & - & - & Low & - \\
\hline- & + & - & + & - & - & + & Low & - \\
\hline - & + & - & + & - & - & - & Low & - \\
\hline- & + & - & + & - & - & + & Low & - \\
\hline- & + & - & - & - & - & - & Low & - \\
\hline- & + & - & - & - & - & - & Low & - \\
\hline - & + & - & - & - & - & - & Low & - \\
\hline- & + & + & - & - & - & - & Low & + \\
\hline- & + & - & - & - & - & + & Low & - \\
\hline - & - & + & + & - & + & + & High & - \\
\hline- & - & - & - & - & - & - & Low & - \\
\hline- & + & - & - & - & + & + & High & - \\
\hline - & + & - & - & - & - & + & High & - \\
\hline- & + & + & - & - & + & - & High & - \\
\hline- & - & - & + & - & + & + & High & + \\
\hline- & + & - & + & - & + & + & High & - \\
\hline- & + & - & + & - & + & - & High & - \\
\hline- & + & - & + & - & + & - & High & - \\
\hline - & + & - & + & - & - & + & High & - \\
\hline- & + & - & - & - & - & - & High & - \\
\hline- & + & - & - & - & - & - & Low & - \\
\hline- & + & - & - & - & - & - & Low & - \\
\hline - & + & - & - & - & - & + & Low & - \\
\hline- & + & - & + & - & + & + & High & - \\
\hline- & + & - & - & - & - & + & High & - \\
\hline- & + & - & + & - & + & + & High & - \\
\hline- & + & - & - & - & + & + & High & - \\
\hline - & + & - & - & - & - & - & Low & - \\
\hline- & + & - & - & - & - & - & Low & - \\
\hline- & + & - & - & - & - & - & Low & - \\
\hline- & + & - & - & - & - & - & High & - \\
\hline- & + & - & + & - & + & + & High & - \\
\hline- & + & + & + & - & + & - & High & - \\
\hline- & + & - & - & - & - & - & Low & + \\
\hline
\end{tabular}




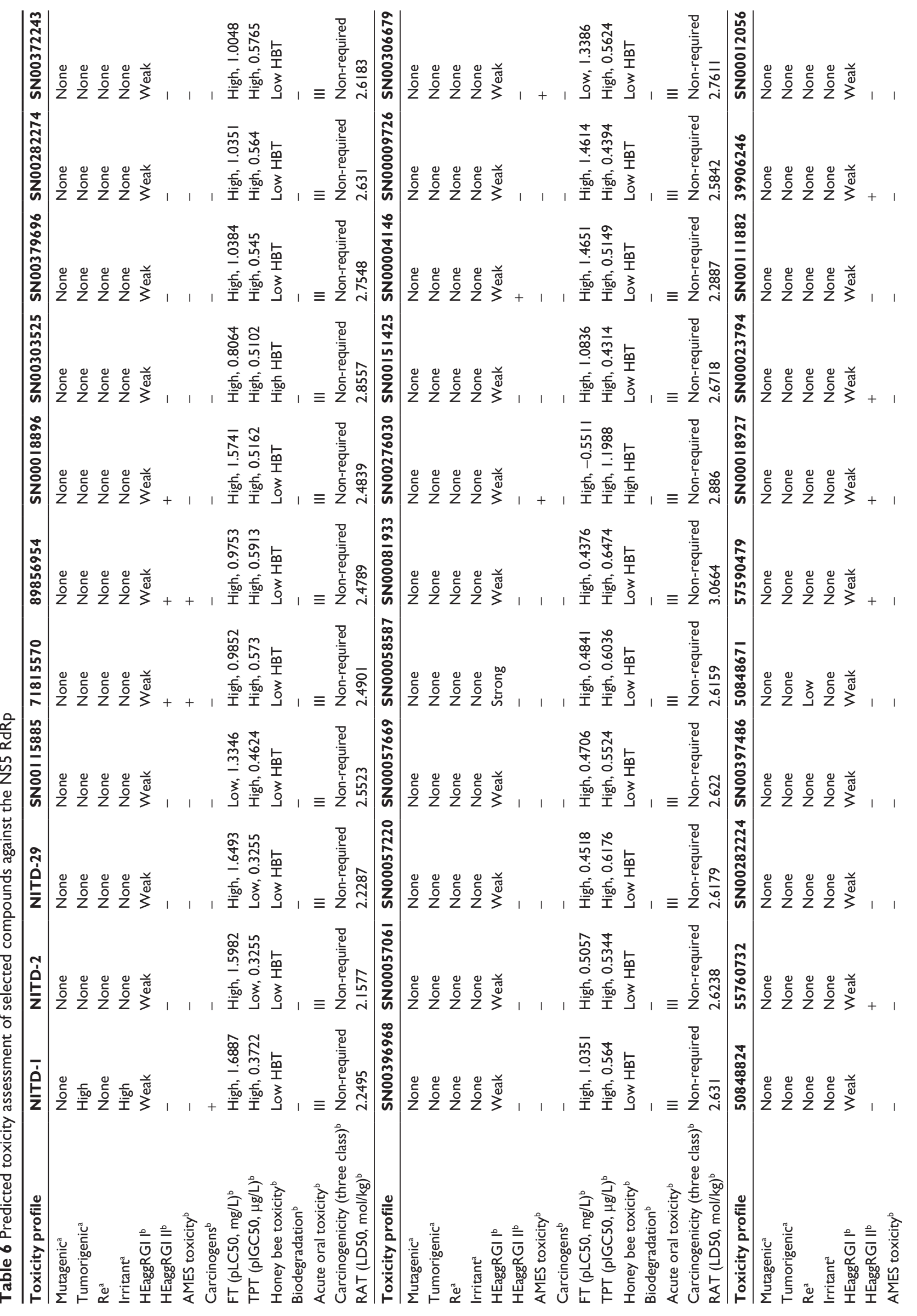




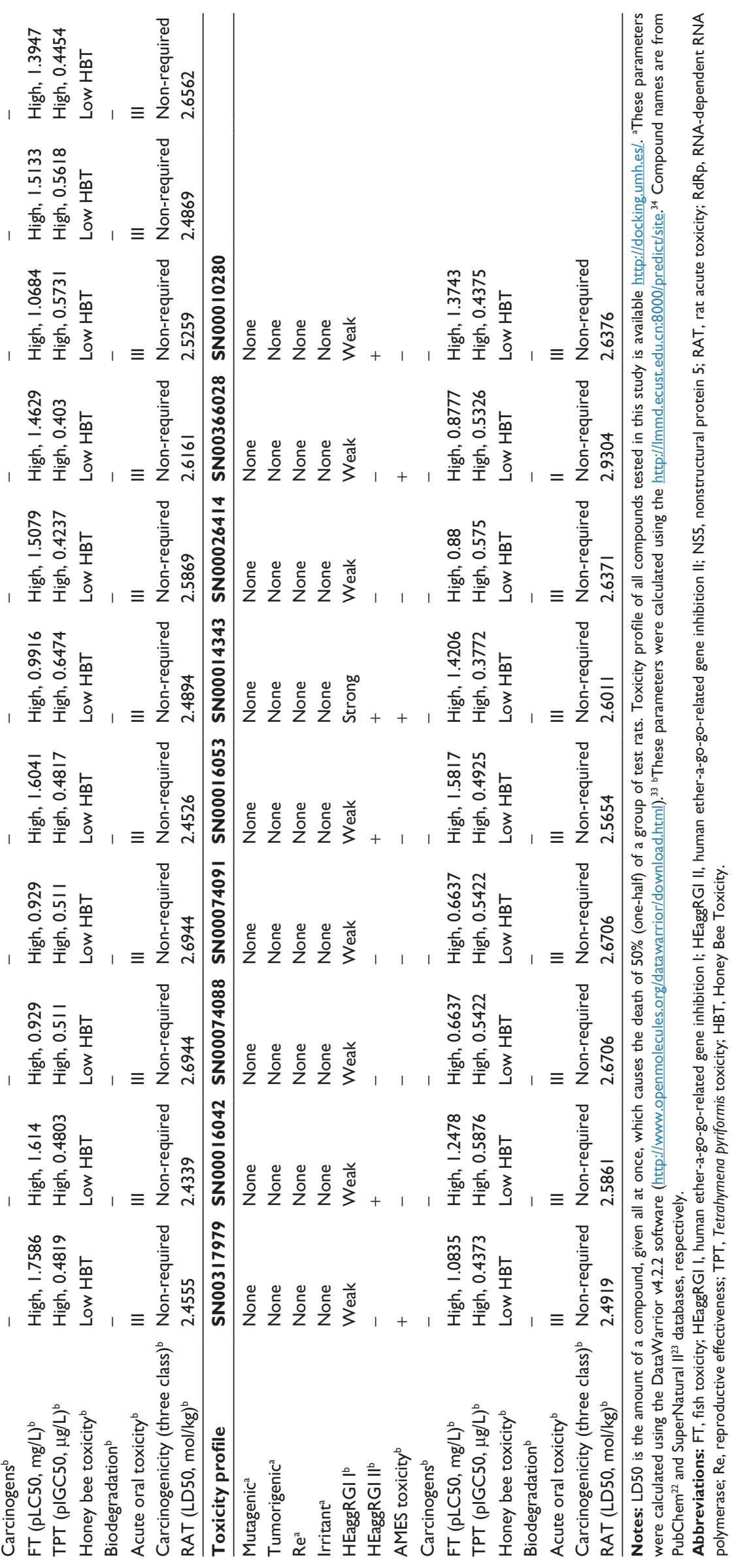




\section{Acknowledgments}

We thank the "Fundación del Centro de Supercomputación de Castilla y León (FCSCL)" for allowing us to use their facilities and resources on the Linux cluster calendula.fcsc.es. We are grateful to Research, Technological Innovation and Supercomputing Center of Extremadura (CenitS) and COMPUTAEX for allowing us to use the supercomputing facilities (LUSITANIA II) and for the support provided to us. Also, we thank the computing center of the Extremadura Research Centre for Advanced Technologies (CETA-CIEMAT), funded by the European Regional Development Fund. CETA-CIEMAT belongs to CIEMAT and the Government of Spain. We thank the editor and three anonymous reviewers for their constructive comments, which helped us to improve the manuscript. This work was partly supported by grants AGL2015-67995-C3-1-R and AGL2014-51773-C3-1-R from the Spanish MICINN, and PROMETEO/2016/006 grant from Generalitat Valenciana.

\section{Disclosure}

The authors report no conflicts of interest in this work.

\section{References}

1. Weissenbock H, Hubalek Z, Bakonyi T, Nowotny N. Zoonotic mosquito-borne flaviviruses: worldwide presence of agents with proven pathogenicity and potential candidates of future emerging diseases. Vet Microbiol. 2010;140(3-4):271-280.

2. Papageorgiou L, Loukatou S, Sofia K, Maroulis D, Vlachakis D. An updated evolutionary study of Flaviviridae NS3 helicase and NS5 RNA-dependent RNA polymerase reveals novel invariable motifs as potential pharmacological targets. Mol Biosyst. 2016;2(7):2080-2093.

3. International Committee on Taxonomy of Viruses, King AMQ. Virus Taxonomy: Classification and Nomenclature of Viruses Ninth Report of the International Committee on Taxonomy of Viruses. London: Academic Press; 2012.

4. Brady OJ, Gething PW, Bhatt S, et al. Refining the global spatial limits of dengue virus transmission by evidence-based consensus. PLoS Negl Trop Dis. 2012;6(8):e1760.

5. Shepard DS, Undurraga EA, Halasa YA, Stanaway JD. The global economic burden of dengue: a systematic analysis. Lancet Infect Dis. 2016;16(8):935-941.

6. Alagarasu K. Introducing dengue vaccine: implications for diagnosis in dengue vaccinated subjects. Vaccine. 2016;34(25):2759-2761.

7. Mackenzie J. Wrapping things up about virus RNA replication. Traffic. 2005;6(11):967-977.

8. Paul D, Bartenschlager R. Flaviviridae replication organelles: oh, what a tangled web we weave. Annu Rev Virol. 2015;2(1):289-310.

9. Issur M, Geiss BJ, Bougie I, et al. The flavivirus NS5 protein is a true RNA guanylyltransferase that catalyzes a two-step reaction to form the RNA cap structure. RNA. 2009;15(12):2340-2350.

10. Decroly E, Ferron F, Lescar J, Canard B. Conventional and unconventional mechanisms for capping viral mRNA. Nat Rev Microbiol. 2012;10(1):51-65.

11. Zhao Y, Soh TS, Zheng J, et al. A crystal structure of the Dengue virus NS5 protein reveals a novel inter-domain interface essential for protein flexibility and virus replication. PLoS Pathog. 2015;11(3):e1004682.
12. Yap TL, Xu T, Chen YL, et al. Crystal structure of the dengue virus RNA-dependent RNA polymerase catalytic domain at 1.85 -angstrom resolution. J Virol. 2007;81(9):4753-4765.

13. Bartholomeusz A, Thompson P. Flaviviridae polymerase and RNA replication. J Viral Hepat. 1999;6(4):261-270.

14. You S, Falgout B, Markoff L, Padmanabhan R. In vitro RNA synthesis from exogenous dengue viral RNA templates requires long range interactions between 5 '- and 3'-terminal regions that influence RNA structure. J Biol Chem. 2001;276(19):15581-15591.

15. Malet H, Masse N, Selisko B, et al. The flavivirus polymerase as a target for drug discovery. Antiviral Res. 2008;80(1):23-35.

16. De Clercq E, Neyts J. Antiviral agents acting as DNA or RNA chain terminators. Handb Exp Pharmacol. 2009(189):53-84.

17. De Francesco R, Tomei L, Altamura S, Summa V, Migliaccio G. Approaching a new era for hepatitis $\mathrm{C}$ virus therapy: inhibitors of the NS3-4A serine protease and the NS5B RNA-dependent RNA polymerase. Antiviral Res. 2003;58(1):1-16.

18. Yin Z, Chen YL, Schul W, et al. An adenosine nucleoside inhibitor of dengue virus. Proc Natl Acad Sci U S A. 2009;106(48):20435-20439.

19. Kohler JJ, Lewis W. A brief overview of mechanisms of mitochondrial toxicity from NRTIs. Environ Mol Mutagen. 2007;48(3-4):166-172.

20. Behnam MA, Nitsche C, Boldescu V, Klein CD. The medicinal chemistry of dengue virus. $J$ Med Chem. 2016;59(12):5622-5649.

21. Niyomrattanakit $\mathrm{P}$, Chen $\mathrm{YL}$, Dong $\mathrm{H}$, et al. Inhibition of dengue virus polymerase by blocking of the RNA tunnel. $J$ Virol. 2010;84(11): $5678-5686$.

22. Wang Y, Xiao J, Suzek TO, Zhang J, Wang J, Bryant SH. PubChem: a public information system for analyzing bioactivities of small molecules. Nucleic Acids Res. 2009;37(Web Server issue):W623-W633.

23. Banerjee P, Erehman J, Gohlke BO, Wilhelm T, Preissner R, Dunkel M. Super Natural II - a database of natural products. Nucleic Acids Res. 2015; 43(Database issue):D935-D939.

24. Zhao Y, Soh TS, Lim SP, et al. Molecular basis for specific viral RNA recognition and 2'-O-ribose methylation by the dengue virus nonstructural protein 5 (NS5). Proc Natl Acad Sci U S A. 2015;112(48): 14834-14839.

25. Noble CG, Lim SP, Chen YL, et al. Conformational flexibility of the Dengue virus RNA-dependent RNA polymerase revealed by a complex with an inhibitor. $J$ Virol. 2013;87(9):5291-5295.

26. Lim SP, Koh JH, Seh CC, et al. A crystal structure of the dengue virus non-structural protein 5 (NS5) polymerase delineates interdomain amino acid residues that enhance its thermostability and de novo initiation activities. J Biol Chem. 2013;288(43):31105-31114.

27. Biasini M, Bienert S, Waterhouse A, et al. SWISS-MODEL: modelling protein tertiary and quaternary structure using evolutionary information. Nucleic Acids Res. 2014;42(Web Server issue):W252-W258.

28. Encinar JA, Fernandez-Ballester G, Galiano-Ibarra V, Micol V. In silico approach for the discovery of new PPARgamma modulators among plant-derived polyphenols. Drug Des Devel Ther. 2015;9: $5877-5895$.

29. Morris GM, Huey R, Lindstrom W, et al. AutoDock4 and AutoDockTools4: automated docking with selective receptor flexibility. J Comput Chem. 2009;30(16):2785-2791.

30. Schymkowitz J, Borg J, Stricher F, Nys R, Rousseau F, Serrano L. The FoldX web server: an online force field. Nucleic Acids Res. 2005; 33(Web Server issue):W382-W388.

31. Trott O, Olson AJ. AutoDock Vina: improving the speed and accuracy of docking with a new scoring function, efficient optimization, and multithreading. J Comput Chem. 2010;31(2):455-461.

32. Lipinski CA, Lombardo F, Dominy BW, Feeney PJ. Experimental and computational approaches to estimate solubility and permeability in drug discovery and development settings. Adv Drug Deliv Rev. 2001; 46(1-3):3-26.

33. Sander T, Freyss J, von Korff M, Rufener C. DataWarrior: an opensource program for chemistry aware data visualization and analysis. $J$ Chem Inf Model. 2015;55(2):460-473. 
34. Cheng F, Li W, Zhou Y, et al. admetSAR: a comprehensive source and free tool for assessment of chemical ADMET properties. J Chem Inf Model. 2012;52(11):3099-3105.

35. Meng XY, Zhang HX, Mezei M, Cui M. Molecular docking: a powerful approach for structure-based drug discovery. Curr Comput Aided Drug Des. 2011;7(2):146-157.

36. Jaghoori MM, Bleijlevens B, Olabarriaga SD. 1001 Ways to run AutoDock Vina for virtual screening. J Comput Aided Mol Des. 2016; 30(3):237-249.

37. Moroy G, Martiny VY, Vayer P, Villoutreix BO, Miteva MA. Toward in silico structure-based ADMET prediction in drug discovery. Drug Discov Today. 2012;17(1-2):44-55.
38. Merlot C. Computational toxicology - a tool for early safety evaluation. Drug Discov Today. 2010;15(1-2):16-22.

39. Zhao YH, Abraham MH, Le J, et al. Rate-limited steps of human oral absorption and QSAR studies. Pharm Res. 2002;19(10):1446-1457.

40. Anzenbacher P, Anzenbacherova E. Cytochromes P450 and metabolism of xenobiotics. Cell Mol Life Sci. 2001;58(5-6):737-747.

41. Roche O, Trube G, Zuegge J, Pflimlin P, Alanine A, Schneider G. A virtual screening method for prediction of the HERG potassium channel liability of compound libraries. Chembiochem. 2002;3(5): 455-459.

\section{Publish your work in this journal}

Drug Design, Development and Therapy is an international, peerreviewed open-access journal that spans the spectrum of drug design and development through to clinical applications. Clinical outcomes, patient safety, and programs for the development and effective, safe, and sustained use of medicines are the features of the journal, which has also been accepted for indexing on PubMed Central. The manuscript management system is completely online and includes a very quick and fair peer-review system, which is all easy to use. Visit http://www.dovepress.com/testimonials.php to read real quotes from published authors.

Submit your manuscript here: http://www.dovepress.com/drug-design-development-and-therapy-journal 